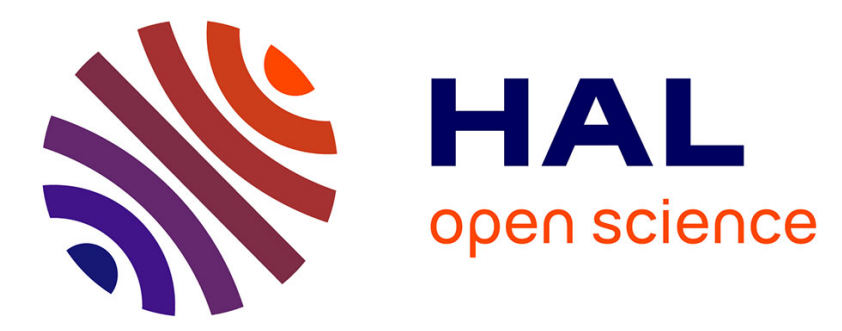

\title{
The First Ultraviolet Detection of the Large Magellanic Cloud Pulsar PSR B0540-69 and Its Multi-wavelength Properties
}

\author{
R.P. Mignani, A. Shearer, A. de Luca, F.E. Marshall, L. Guillemot, D.A. \\ Smith, B. Rudak, L. Zampieri, C. Barbieri, G. Naletto, et al.
}

\section{To cite this version:}

R.P. Mignani, A. Shearer, A. de Luca, F.E. Marshall, L. Guillemot, et al.. The First Ultraviolet Detection of the Large Magellanic Cloud Pulsar PSR B0540-69 and Its Multi-wavelength Properties. Astrophys.J., 2019, 871 (2), pp.246. 10.3847/1538-4357/aafb04 . hal-01909181

\section{HAL Id: hal-01909181 https://hal.science/hal-01909181}

Submitted on 16 Apr 2020

HAL is a multi-disciplinary open access archive for the deposit and dissemination of scientific research documents, whether they are published or not. The documents may come from teaching and research institutions in France or abroad, or from public or private research centers.
L'archive ouverte pluridisciplinaire HAL, est destinée au dépôt et à la diffusion de documents scientifiques de niveau recherche, publiés ou non, émanant des établissements d'enseignement et de recherche français ou étrangers, des laboratoires publics ou privés. 


\title{
The First Ultraviolet Detection of the Large Magellanic Cloud Pulsar PSR B0540-69 and Its Multi-wavelength Properties
}

\author{
R. P. Mignani ${ }^{1,2}$ (D), A. Shearer ${ }^{3}$, A. de Luca $^{1}$, F. E. Marshall ${ }^{4}$, L. Guillemot ${ }^{5}$ (D), D. A. Smith ${ }^{6}$, B. Rudak ${ }^{7}$ (D), L. Zampieri ${ }^{8}$ (D), \\ C. Barbieri ${ }^{8,9,10}$, G. Naletto ${ }^{9}$, C. Gouiffes ${ }^{11}$, and G. Kanbach ${ }^{12}$ \\ ${ }^{1}$ INAF-Istituto di Astrofisica Spaziale e Fisica Cosmica Milano, via E. Bassini 15, I-20133, Milano, Italy \\ 2 Janusz Gil Institute of Astronomy, University of Zielona Góra, ul Szafrana 2, 65-265, Zielona Góra, Poland \\ ${ }^{3}$ Centre for Astronomy, School of Physics, National University of Ireland Galway, University Road, Galway, Ireland \\ ${ }^{4}$ Astrophysics Science Division, NASA Goddard Space Flight Center, Greenbelt, MD 20771, USA \\ ${ }^{5}$ Laboratoire de Physique et Chimie de l'Environnement et de l'Espace (LPC2E), CNRS-Université d'Orléans, F-45071 Orléans, France \\ ${ }^{6}$ Centre d'Etudes Nucléaires de Bordeaux Gradignan, IN2P3/CNRS, Université de Bordeaux 1, BP120, F-33175, Gradignan Cedex, France \\ ${ }^{7}$ Nicolaus Copernicus Astronomical Center, Polish Academy of Sciences, ul. Rabiańska 8, 87100, Toruń, Poland \\ ${ }^{8}$ INAF-Osservatorio Astronomico di Padova, Vicolo dell'Osservatorio 5, I-35122 Padova, Italy \\ 9 Department of Physics and Astronomy "G. Galilei," University of Padova, Vic. Osservatorio 3, I-35122 Padova, Italy \\ ${ }^{10}$ Centro di Ateneo di Studi ed Attivitá Spaziali "Giuseppe Colombo," University of Padova, Via Venezia 15, I-35131 Padova, Italy \\ ${ }^{11}$ Laboratoire AIM, UMR 7158 (CEA/Irfu, CNRS/INSU, Université Paris VII), CEA Saclay, Bt. 709, F-91191 Gif-sur-Yvette Cedex, France \\ ${ }_{12}$ Max-Planck Institut für Extraterrestrische Physik, Giessenbachstrasse 1, D-85741, Garching bei München, Germany \\ Received 2018 September 27; revised 2018 December 23; accepted 2018 December 25; published 2019 February 5
}

\begin{abstract}
We observed the young ( $\sim 1700 \mathrm{yr}$ ) pulsar PSR B0540-69 in the ultraviolet for the first time with the Space Telescope Imaging Spectrograph on board the Hubble Space Telescope. Imaging observations with the NUVMAMA and ultraviolet FUV-MAMA detectors in TIME-TAG mode allowed us to clearly detect the pulsar in two bands around 2350 and $1590 \AA$, with magnitudes $m_{\mathrm{NUV}}=21.45 \pm 0.02$ and $m_{\mathrm{FUV}}=21.83 \pm 0.10$. We also detected the pulsar wind nebula in the NUV-MAMA image, with a morphology similar to that observed in the optical and near-infrared (IR). The extinction-corrected NUV and FUV pulsar fluxes are compatible with a very steep power-law spectrum $F_{\nu} \propto \nu^{-\alpha}$ with spectral index $\alpha_{\mathrm{UV}} \sim 3$, and incompatible with a Rayleigh-Jeans spectrum, indicating a non-thermal origin of the emission. The comparison with the optical/near-IR power-law spectrum (spectral index $\alpha_{\mathrm{O}, \mathrm{nIR}} \sim 0.7$ ), indicates an abrupt turnover at wavelengths below $2500 \AA$, not yet observed in other pulsars. We detected pulsations in both the NUV and FUV data at the $50 \mathrm{~ms}$ pulsar period. In both cases, the folded light curve features a broad pulse with two peaks closely spaced in phase, as observed in the optical and X-ray light curves. The NUV/FUV peaks are also aligned in phase with those observed in the radio (1.4 GHz), optical, X-ray, and $\gamma$-ray light curves, as in the Crab pulsar, implying a similar beaming geometry across all wavelengths. PSR B0540-69 is now the fifth isolated pulsar, together with Crab, Vela, PSR B0656+14, and the radio-quiet Geminga, detected in the optical, UV, near-IR, X-rays, and $\gamma$-rays, and seen to pulsate in at least four of these energy bands.
\end{abstract}

Key words: pulsars: general - pulsars: individual (PSR B0540-69)

\section{Introduction}

Pulsars are rapidly spinning neutron stars that emit electromagnetic radiation (mostly) at the expenses of their rotational energy (Gold 1968; Pacini 1968), and hence are also referred to as rotation-powered pulsars. Apart from the radio band, where the first of the over 2500 radio pulsars known to date $^{13}$ was originally discovered (Hewish et al. 1968), pulsars are also observed in X-rays, $\gamma$-rays, optical, infrared (IR), ultraviolet (UV), and the submillimeter region (Mignani et al. 2017).

Owing to their intrinsic faintness, the number of pulsar detections at optical energies by and large lags behind that at high energies. After the Crab pulsar (PSR B0531+21; Cocke et al. 1969), which was the first one identified through its optical pulsations at the radio period (Cocke et al. 1969), only eight isolated pulsars (i.e., not in binary systems) have been firmly identified in the optical plus two candidates (see Mignani 2011 for a review), and three more identifications have recently been proposed (Moran et al. 2013; Mignani et al. 2016b; Rangelov et al. 2017). Optical pulsations have been detected

\footnotetext{
${ }^{13}$ See ATNF pulsar catalog (Manchester et al. 2005).
}

only for some of them, though. Indeed, apart from the Crab (Cocke et al. 1969), optical pulsations have been detected for only four other pulsars: the Vela pulsar (PSR B0833-45; Wallace et al. 1977), PSR B0540-69 (Middleditch \& Pennypacker 1985), PSR B0656+14 (Shearer et al. 1997), and Geminga (Shearer et al. 1998). Eight of the isolated pulsars identified in the optical have also been detected in the $\mathrm{UV}^{14}$ with the Hubble Space Telescope (HST) and four of them (Crab, Vela, PSR B0656+14, and Geminga) pulsate in the UV (Percival et al. 1993; Kargaltsev et al. 2005; Romani et al. 2005; Shibanov et al. 2005), besides the optical band. These four pulsars have also been identified in the near-IR (Mignani et al. 2012 and references therein), but pulsations in this band have been detected only for the Crab (e.g., Eikenberry et al. 1997).

The UV/optical/near-IR (hereafter UVOIR) spectra of young pulsars $\left(\tau_{\mathrm{C}} \lesssim 10 \mathrm{kyr}\right.$ ), where $\tau_{\mathrm{C}}$ is the characteristic age, ${ }^{15}$ show the signature of non-thermal, likely synchrotron, emission from

\footnotetext{
${ }^{14}$ For simplicity, from this point on the term UV is meant to encompass the near, middle and far UV, but not the extreme UV.

15 This is defined as $P_{\mathrm{s}} /\left(2 \dot{P}_{\mathrm{s}}\right)$, where $P_{\mathrm{s}}$ and $\dot{P}_{\mathrm{s}}$ are the pulsar spin period and its first derivative, respectively.
} 
the neutron star magnetosphere (see, e.g., Mignani 2011), characterized by power-law (PL) spectra $F_{\nu} \propto \nu^{-\alpha}(\alpha \sim 0-1)$. Differences in the spectral index $\alpha$ across the three bands are observed in some cases, e.g., the Crab pulsar (Sollerman 2003), but not in others, e.g., the Vela pulsar (Zyuzin et al. 2013). In middle-aged pulsars $\left(\tau_{\mathrm{C}} \approx 0.1-1 \mathrm{Myr}\right)$, a second emission component is present in the optical/UV, associated with thermal emission from the neutron star surface and characterized by a Rayleigh-Jeans (RJ) spectrum with a brightness temperature $T_{\mathrm{B}} \approx 10^{5} \mathrm{~K}$ (Mignani 2011).

Despite the optical and UV being very close in wavelength, differences exist in the pulsar light curves. ${ }^{16}$ In particular, HST observations showed that the widths and separations of the two peaks in the Crab light curve (known as the Main Pulse and Interpulse) are larger in the optical than in the UV (Percival et al. 1993), which is perhaps related to the difference in the PL slope between these two bands (Sollerman 2003). In the case of Vela possible differences in the widths and separations of the two main peaks between the optical and the UV light curves cannot be appreciated owing to the lower statistics, although they differ in the structure of the smaller peaks (Romani et al. 2005). At variance with the Crab, there is no difference in the PL slope from the optical to the UV (Zyuzin et al. 2013). HST observations of the middle-aged pulsars PSR B0656+14 (Shibanov et al. 2005) and Geminga (Kargaltsev et al. 2005) also showed differences in their light curves from the optical to the UV. This might also be due to the rising contribution of the RJ component in the UV relative to the PL component, with extra modulations possibly produced by hot spots on the neutron star surface. Detecting more optical/UV pulsars is important for study of the evolution of the light curve and spectrum across these two bands and to infer the characteristics and geometry of the corresponding emission regions.

PSR B0540-69 in the Large Magellanic Cloud (LMC) is the second brightest optical pulsar $(V=22.5)$ after the Crab and an obvious target for UV observations, which have thus far not been performed. It is referred to as the Crab "twin" because it is very similar in spin period $\left(P_{\mathrm{s}}=50 \mathrm{~ms}\right)$, period derivative $\left(\dot{P}_{\mathrm{s}} \sim 4.78 \times 10^{-13} \mathrm{~s} \mathrm{~s}^{-1}\right)$, characteristic age $\left(\tau_{\mathrm{C}} \sim 1.7 \mathrm{kyr}\right)$, rotational energy loss $\left(\dot{E} \sim 1.5 \times 10^{38} \mathrm{erg} \mathrm{s}^{-1}\right)$, and surface magnetic field $\left(B_{\mathrm{s}} \sim 4.98 \times 10^{12} \mathrm{G}\right){ }^{17}$

The LMC distance $(48.97 \pm 0.09 \mathrm{kpc}$; Storm et al. 2011) makes PSR B0540-69 one of the faintest radio pulsars (Manchester et al. 1993). Indeed, it was discovered in X-rays (Seward et al. 1984), becoming the first extragalactic pulsar detected at any wavelength. PSR B0540-69 is the latest pulsar to have been detected in the near-IR (Mignani et al. 2012) and has also been recently detected as a $\gamma$-ray pulsar (Fermi LAT Collaboration et al. 2015) by the Fermi Large Area Telescope (LAT). Like other young pulsars (Kargaltsev et al. 2017), it is embedded in a bright pulsar wind nebula (PWN) visible from the near-IR to the soft/hard X-rays (Petre et al. 2007; Słowikowska et al. 2007; Mignani et al. 2012). Optical pulsations were detected by Middleditch \& Pennypacker (1985), while the pulsar counterpart was later identified via high-resolution imaging (Caraveo et al. 1992; Shearer et al. 1994). The optical light curve (Middleditch et al. 1987;

\footnotetext{
16 Through the text we implicitly refer to the light curves folded at the pulsar spin period.

${ }^{17}$ The latter two values have been derived from the standard formulae $\dot{E}=4 \times 10^{46} \dot{P}_{\mathrm{s}} / P_{\mathrm{s}}^{3} \mathrm{erg} \mathrm{s}^{-1}$ and $B=3.2 \times 10^{19} \sqrt{P_{\mathrm{s}} \dot{P}_{\mathrm{s}}} \mathrm{G}$, derived by assuming for the neutron star a moment of inertia $I=10^{45} \mathrm{~g} \mathrm{~cm}^{2}$.
}

Gouiffes et al. 1992; Boyd et al. 1995) features a broad pulse, which is actually resolved into two peaks (see also Gradari et al. 2011).

The optical spectrum of PSR B0540-69 is characterized by a PL (e.g., Serafimovich et al. 2004). The measurement of significant phase-averaged polarization with the HST (Mignani et al. 2010b; Lundqvist et al. 2011) confirmed the magnetospheric origin of its optical emission. HST and Very Large Telescope (VLT) adaptive optics images (Mignani et al. 2010b, 2012) clearly resolved PSR B0540-69 from its compact (4") PWN, making it possible to precisely measure the pulsar flux. This yielded the most accurate measurement of its PL spectral index in the optical/near-IR $\left(\alpha_{\mathrm{O}, \mathrm{nIR}}=0.70 \pm 0.04\right)$, which is similar to that in X-rays $\left(\alpha_{\mathrm{X}}=0.83 \pm 0.13\right)$, measured from Chandra spectroscopy (Kaaret et al. 2001). However, the optical fluxes fall below the extrapolation of the X-ray PL (Mignani et al. 2010b), suggesting a spectral flattening in the UV. Determining the pulsar spectrum in the UV, then, is key to confirming the expected flattening, whereas measuring the UV light curve is key to determining whether such flattening is associated with different optical and UV light curve profiles, as possibly observed in the Crab pulsar (Percival et al. 1993).

Here, we present the results of the first UV observations of PSR B0540-69, carried out with the HST. This manuscript is organized as follows: observations and data analysis are described in Section 2, and the results are presented and discussed in Sections 3 and 4, respectively. Summary and conclusions follow in Section 5.

\section{Observations}

\subsection{Description of Observations}

We observed PSR B0540-69 with the HST during Cycle 23 (Prog. ID: 14250; PI: Mignani) on 2017 February 27 and 28, as part of the UV Initiative Program. We used the Space Telescope Imaging Spectrograph (STIS) and collected data with both its NUV- and FUV-MAMA (Multi-Anode Microchannel Array) detectors, which are sensitive in the spectral ranges $1600-3100 \AA$ and $1150-1700 \AA$, respectively.

The detectors were operated in imaging $\left(25^{\prime \prime} \times 25^{\prime \prime}\right.$ field of view) TIME-TAG mode, chosen for two principal reasons: (i) to clearly resolve the pulsar emission from that of the surrounding PWN ( $\sim 4^{\prime \prime}$ diameter), thanks to a spatial resolution of 0. ! $024 /$ pixel, and (ii) to search for pulsations at the pulsar period $(50 \mathrm{~ms})$ and accurately sample the light curve, thanks to a time resolution of $125 \mu \mathrm{s}$. For the NUV- and FUVMAMA detectors we used their F25QTZ filters, ${ }^{18}$ which have central wavelengths and FWHM bandwidths of $\lambda=2359.3 \AA$, $\Delta \lambda=998.7 \AA$ and $\lambda=1596.2 \AA, \Delta \lambda=231.6 \AA$, respectively (Riley 2017). These combinations provide high-throughput broadband UV imaging, minimizing the background contribution from geocoronal emission lines and maximizing the spectral coverage achievable with the STIS MAMAs.

The planned exposures were allocated in six spacecraft orbits, equally distributed between the NUV and FUV observations (three orbits each), and split into two different visits to cope with the HST scheduling constraints. The same roll angle of $32^{\circ} .4934$ (measured east of north) was used in both

\footnotetext{
18 Since the filters have the same name for both MAMA detectors, hereafter we simply distinguish the two data sets by the detector name (NUV and FUV for short).
} 

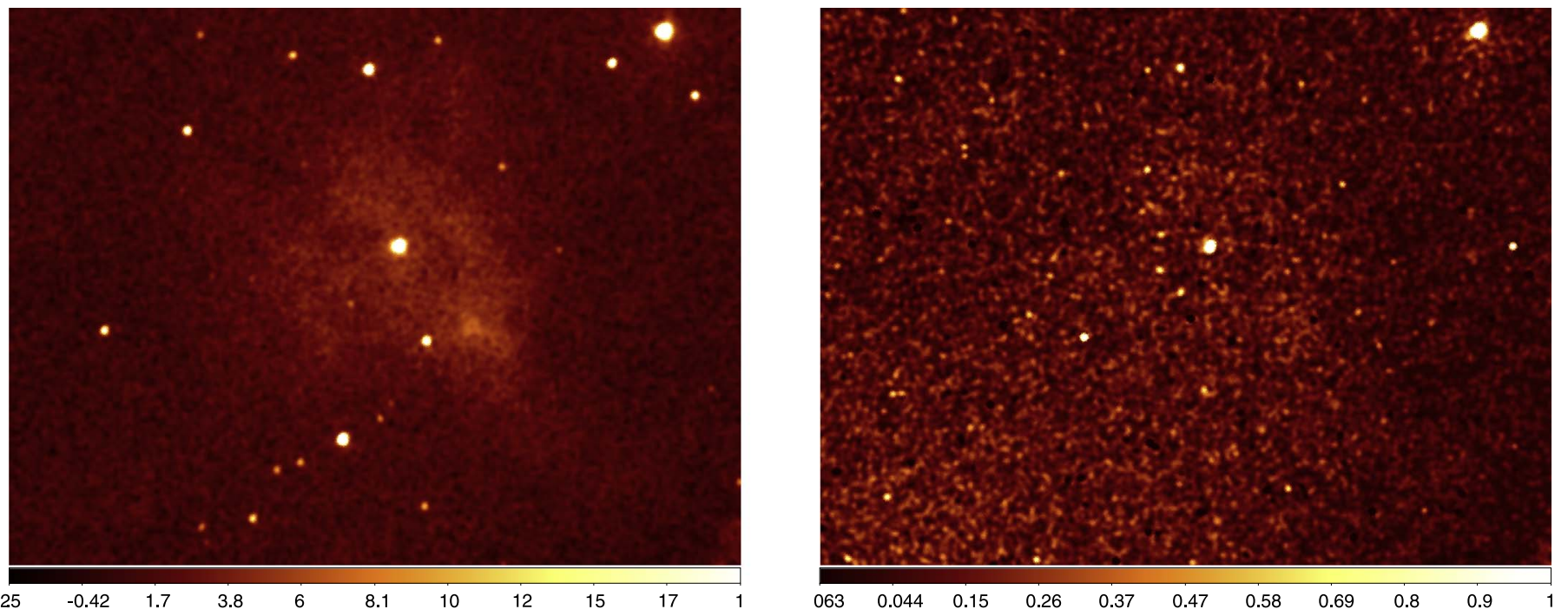

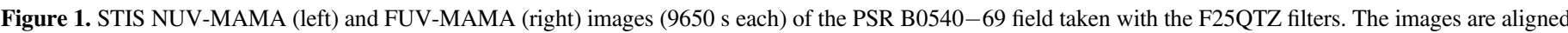

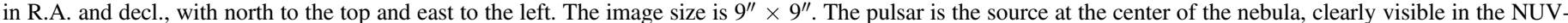

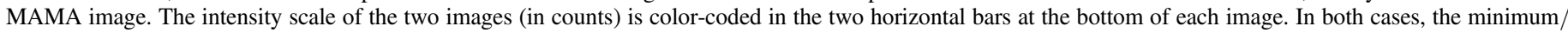

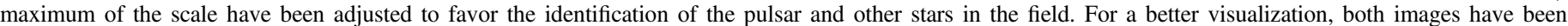
smoothed with a Gaussian function using a kernel of 2 pixel radius.

visits. The exposure time per orbit was defined to fully exploit the target visibility window. ${ }^{19}$ After accounting for instrument overheads and guide star acquisitions, we acquired one $3050 \mathrm{~s}$ and two $3300 \mathrm{~s}$ exposures (1000 s buffer time) in each visit for a net total integration time of $9650 \mathrm{~s}$ for both the NUV and FUV observations.

\subsection{Data Analysis}

We retrieved our data from the Mikulski Archive for Space Telescopes ${ }^{20}$ after routine data reduction and calibration steps were applied through the CALSTIS pipeline under the STSDAS package. These steps are: dark subtraction, flat-fielding, corrections for geometric distortion and detector nonlinearity, and flux calibration, which have all been implemented using the calibration frames and tables closest in time to our observations. In order to increase the signal-to-noise ratio, for each data set we then co-added the single exposures using the STSDAS task combine, which also applies rejection of cosmic-ray hits.

We checked the astrometry of the NUV and FUV images, determined by the HST aspect solution, against the HST/ WFPC2 images of Mignani et al. (2010b), whose astrometry was recalibrated in the reference frame of the Two Micron All Sky Survey (Skrutskie et al. 2006) with an overall accuracy of 0 ". 12-0." 15. To account for the measured absolute offsets of $\sim 0$." 23 and $\sim 0$ ". 57 in the NUV and FUV image astrometry, respectively, we used a grid of reference stars detected in a $\sim 12^{\prime \prime}$ radius around PSR B0540-69 to register the MAMA images onto the astrometry reference frame of the WFPC2 images with an accuracy of better than 0 ". 01 . We used the NUV and FUV images with the recalibrated astrometry as a reference for the pulsar identification.

\footnotetext{
$\overline{19}$ Owing to scheduling constraints in Cycle 23 it was not possible to observe our target in Continuous Viewing Zone and the maximum visibility window before Earth occultation was about $3500 \mathrm{~s}$ per orbit.

${ }^{20}$ https://archive.stsci.edu/hst/search.php
}

\section{Results}

\subsection{Imaging and Photometry}

Figure 1 shows the NUV- and FUV-MAMA images of the PSR B0540-69 field obtained after the processing described in the previous section. We clearly detected PSR B0540-69 in both the NUV and FUV images at a position coincident with its optical coordinates computed by Mignani et al. (2010b): $\alpha_{\mathrm{J} 2000}=$ $05^{\mathrm{h}} 40^{\mathrm{m}} 11^{\mathrm{s}} 202(0.009), \delta_{\mathrm{J} 2000}=-69^{\circ} 19^{\prime} 54^{\prime \prime} 17\left(0{ }^{\prime \prime} 05\right) .^{21}$ Ours is the first detection ever of PSR B0540-69 in the UV, which also makes it the fifth isolated pulsar, among the $\sim 2300$ known, that has been detected in the near-IR, optical, UV, $\mathrm{X}$-rays, and $\gamma$-rays, after the Crab and Vela pulsars, PSR B0656 +14 , and Geminga (the fourth among radio pulsars ${ }^{22}$ ). We also detected the PSR B0540-69 PWN in our NUV image (Figure 1, left), with a structure and extent similar to what is observed in the optical and near-IR (Mignani et al. 2010b, 2012). The PWN is at most barely visible, however, in the FUV image (Figure 1, right). The bright emission knot in the PWN detected at $\sim 1$ !'7 southwest of the pulsar in $H S T /$ WFPC2 images (De Luca et al. 2007) is also visible in the STIS/ NUV-MAMA observation, aligned with the major axis of the PWN. Since our paper is focused on the pulsar, a coherent multi-wavelength spectral and spatial analysis of the PWN and its features will be the subject of a subsequent paper.

For both the NUV and FUV images, we computed the pulsar fluxes through aperture photometry employing the tools in the IRAF $^{23}$ package PHOT. We used an aperture radius of 10 pixel $(0$. .'24) to maximize the signal-to-noise ratio and we sampled the sky background within an annulus of 25 pixel inner radius

\footnotetext{
21 The errors refer to the average of the pulsar coordinates computed on four independent WFPC2 data sets, see Mignani et al. (2010b) for details.

22 Geminga (PSR J0633+1746) has not yet been unambiguously detected as a radio pulsar despite many searches, see Maan (2015) and references therein.

23 IRAF is distributed by the National Optical Astronomy Observatories, which are operated by the Association of Universities for Research in Astronomy, Inc., under cooperative agreement with the National Science Foundation.
} 
$(0$ ". 6$)$ to avoid contamination from the wings of the pulsar point-spread function, which are particularly bright in the NUV-MAMA F25QTZ filter, and of 35 pixel outer radius $(0$ ". 84$)$ to avoid including bright stars close to the pulsar. Since the PWN is the main source of background, which is itself not spatially uniform, we carefully checked that our photometry is not very sensitive to the choice of width of the annulus. We then applied the aperture correction to compute the pulsar count-rates in an infinite aperture using the values of the encircled energy fractions for the chosen radius reported in the STIS Instrument Handbook Version 16.0 (Riley 2017) for the NUV- and FUV-MAMA F25QTZ filters. The aperturecorrected, background-subtracted count-rates (CR) are $1.53 \pm 0.02$ and $0.053 \pm 0.005$ counts $s^{-1}$ for the NUV and FUV images, respectively. The large difference in CR can be visually appreciated by comparison of the two images (Figure 1), which were obtained over the same integration time $(9650 \mathrm{~s})$ and are thus directly comparable to each other. We converted the corresponding instrumental magnitudes into ST magnitudes (STMag) using the photometric calibration parameter PHOTFLAM, which is closest in time to our observations and reported in the image header, according to the definition: STMag $=-2.5 \times \log _{10}(\mathrm{CR} \times$ PHOTFLAM $)-$ 21.10. The observed magnitudes, i.e., uncorrected for the interstellar extinction, are $m_{\mathrm{NUV}}=21.45 \pm 0.02$ and $m_{\mathrm{FUV}}=$ $21.83 \pm 0.10$, where the associated errors are purely statistical.

\subsection{Timing}

We looked for pulsations in the NUV and FUV data of PSR B0540-69 at its $\sim 50 \mathrm{~ms}$ period. We extracted the time series from the event files using an aperture with a radius of 10 pixels (0!'24), which corresponds to $82 \%$ of the pulsar flux (Riley 2017). Then we used the task hstephem in STSDAS to account for the spacecraft position and velocity during the observations, and the IRAF task otimedelay to convert the photon arrival times from the topocentric reference frame to the barycentre of the solar system. As a reference for the pulsar position we used the most precise coordinates known (Mignani et al. 2010b). Since the pulsar has a proper motion of $<1$ mas yr $^{-1}$ (Mignani et al. 2010b) any displacement between the epoch of our STIS observations (MJD 57811) and that of the reference position (MJD 54272) is much smaller than the absolute uncertainty on the pulsar coordinates (70 mas).

We folded the NUV and FUV time series around the expected pulsar period using as a reference the most recent timing solution for PSR B0540-69 (F. E. Marshall et al. 2019, in preparation), obtained by monitoring the evolution of the pulsar period with the X-ray Telescope (XRT; Burrows et al. 2005) aboard the Neil Gehrels Swift Observatory after the change in the spin-down frequency derivative $\dot{\nu}$ occurred between 2011 December 3 and 17 (Marshall et al. 2015). Results from this monitoring program, which started on 2015 February 17, have been presented in Marshall et al. (2016, 2018). In particular, the data set consists of all the XRT observations of PSR B0540-69 and covers the full time span from 2015 February 17 to 2018 March 28 (MJD $57070-58205$ ), for a total of $176.893 \mathrm{ks}$.

The analysis of the data from the XRT used the procedure described in Marshall et al. $(2015,2016)$. All the observations were made using the window timing mode. The data were processed using the software tool xrtpipeline, and events were screened to maximize the signal from the pulsar. Arrival times were corrected to the barycentre of the solar system using the pulsar position from Mignani et al. (2010b) and the JPL Planetary Ephemeris DE-200 (Standish 1982). ${ }^{24}$ Events were folded on multiple candidate periods, and a sine wave was fit to the best folded light curve. The resulting frequencies and phases at the epochs of the observations were then fit with a spin model using a Taylor expansion of spin frequency and its derivatives through $\ddot{\nu}$. Small glitches in the pulsar on MJD 57546 and 57946 were added to the model to produce a good fit for the entire span of observations. The resulting ephemeris was used to produce a folded X-ray light curve of all the events. The updated timing solution, together with a detailed analysis of the evolution of the spin frequency, will be presented in a separate publication (F. E. Marshall et al. 2019, in preparation).

By folding the NUV and FUV data at the expected pulsar period, we found a clear pulsed signal in both the NUV and FUV time series (Figures 2(a) and (b)), albeit at different significance levels owing to the difference in the pulsar count-rate in the two data sets (see Section 3.1). The detection significance for the NUV and FUV pulsations is $\sim 36 \sigma$ and $\sim 6 \sigma$ respectively, which we computed based on the $Z_{n}^{2}$ statistic (Buccheri et al. 1983). We also computed the detection significance based on a $\chi^{2}$ analysis and obtained comparable results, with $\chi^{2}$ values of 2800 (64 degrees of freedom, dof) and 82 (20 dof) for the NUV and FUV light curves, respectively. The final probability was determined with Monte Carlo simulations for the case of non-normal distributions. The detection of the expected periodicity clearly and independently confirms the UV identification of the pulsar, initially based upon position match with the optical coordinates. Therefore, PSR B0540-69 is also the fifth isolated pulsar for which pulsations have been detected in the UV, optical, X-rays, and $\gamma$-rays. A comprehensive crosscomparison of the multi-wavelength light curves and spectra of these five pulsars is beyond the goals of this work and will be reported elsewhere.

Both the folded NUV and FUV light curves feature a broad pulse resolved into two peaks separated by $\sim 0.3$ in phase (Figure 2). The two peaks look more structured in the NUV light curve than in the FUV one, but this is only an effect of the better count statistics and smaller binning. Although similar, the NUV and FUV light curves show some small differences. For instance, in the FUV light curve the second peak seems to be less intense than the first, whereas in the NUV one the intensity of the two peaks is comparable. Obtaining an FUV light curve with an improved signal-to-noise ratio would help to determine whether this difference in the intensity of the two peaks is real or an effect of the low count statistics. A difference between the relative intensity of the two main peaks is also observed, e.g., in the STIS NUV and FUV light curves of the Vela pulsar, where the intensity of the primary peak with respect to the secondary one increases in the FUV (Romani et al. 2005). On the other hand, in the case of the Crab pulsar there is no appreciable difference in the relative intensity of the primary and secondary peaks between the STIS NUV and FUV

\footnotetext{
${ }^{24}$ In this, we followed the prescription of the HEASOFT tool barycorr that recommends DE-200 for the Swift data, which are based on the FK5 reference frame. More recent ephemeris files, such as DE-405, use the ICRS reference frame. According to the notes to the barycorr tool, using DE-405 instead of DE-200 will cause a maximum error of 2 ns for satellites in low Earth orbit, which is completely negligible for PSR B0540-69. Moreover the STIS data analysis threads recommend the use of DE-200.
} 

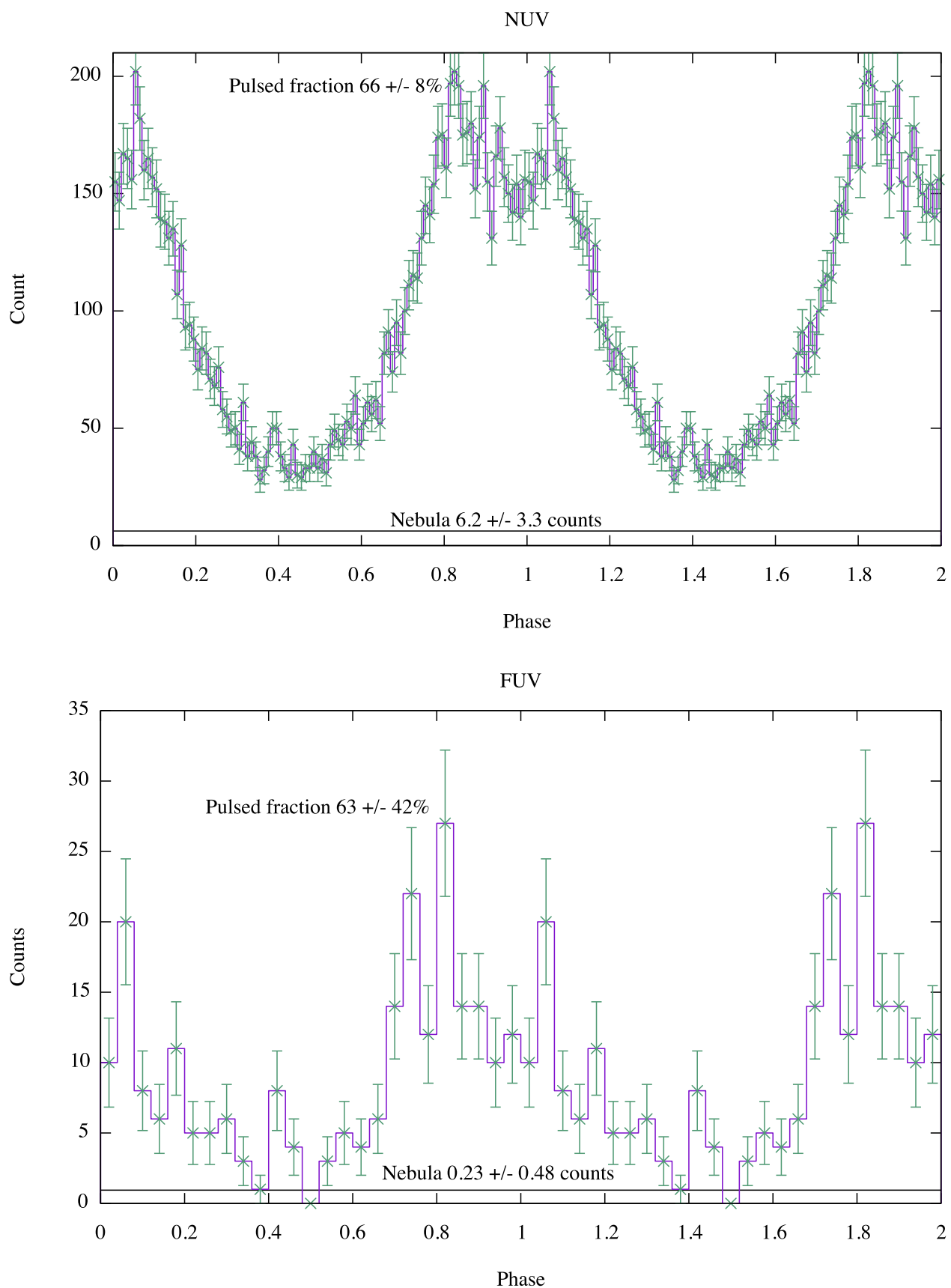

Figure 2. NUV (top) and FUV (bottom) light curves of PSR B0540-69. Two cycles are shown for clarity. Owing to the difference in signal-to-noise ratio, a different phase binning has been applied in each case. The NUV and FUV light curves have been folded using the most recent ephemeris obtained from Swift/XRT observations over the period 2015 February 17-2018 March 28 (F. E. Marshall et al. 2019, in preparation). Pulsar counts have been extracted using an aperture of 10 pixel radius $(0$. " 24$)$. In both panels, the horizontal dashed line marks the background level of the PWN computed in an annulus centered on the pulsar of 25 pixel inner radius and 10 pixel width (Section 4.2).

light curves (Sollerman et al. 2000). A hint of a third peak between the two main ones is visible in the NUV light curve of PSR B0540-69, and it looks more or less prominent depending on the binning. Its estimated significance is $\sim 2 \sigma-3 \sigma$ only and it is even lower in the FUV light curve, where such a third peak is barely visible. A possible precursor to the broad pulse is also visible in the FUV light curve, but is not as clear in the NUV one, indicating that it might be an effect of the different binning. Also in this case, obtaining NUV and FUV light curves with an improved signal-to-noise ratio would help to assess the existence of these features. This complex structure might be reminiscent of that observed in the NUV and FUV light curves of the Vela pulsar, where two small peaks are present in addition to the primary and secondary ones (Romani et al. 2005). At present, however, we cannot determine whether the substructures seen in the light curves of PSR B0540-69 are real or not. Investigations through deeper observations may be worthwhile.

As it can be seen from Figure 2, the signal from PSR B0540 -69 is almost totally pulsed in the NUV and FUV light curves. In particular, the pulsed fraction (PF) is $66 \% \pm 8 \%$ and $63 \% \pm 42 \%$ in the NUV and FUV light curves, respectively, 
where we computed PF from the component above the flux level in the phase interval defined as the off-pulse region, often refereed to as the direct current (DC) level. The DC level is much higher in the NUV light curve than in the FUV one (Figure 2), as expected from the higher PWN background present in the NUV image (Figure 1). This, however, only partially accounts for the DC level, which is well above the nebula background. Indeed, the DC level, calculated in the phase intervals $0.355-0.515$ (NUV) and 0.34-0.54 (FUV), is $35.8 \pm 6.9$ counts and $3.2 \pm 2.8$ counts, respectively. On the other hand, the nebula background, which we sampled in an annulus centered on the pulsar of 25 pixel inner radius and 10 pixel width (see Section 3.1), is only $6.2 \pm 3.3$ counts and $0.23 \pm 0.48$ counts in the NUV and FUV images, respectively.

Such a difference suggests that there is a significant continuous emission component from a source very close to the pulsar, which is not associated with the PWN. A significant DC component is also seen in the light curve of the Crab pulsar and is associated with the unresolved emission from the bright knot in the PWN, at 0." 65 from the pulsar (Słowikowska et al. 2009). No such structure, however, is seen in our high-spatialresolution HST images of PSR B0540-69 (see also Mignani et al. 2010b). Given the small aperture used to extract the pulsar counts ( 0 "! 24 radius), about twice the size of the pointspread function of the image, we deem it unlikely that this DC component is associated with a source other than the pulsar itself. Therefore, there must be an emission component from the pulsar that is not pulsed. This would be the case, for instance, if such a component were emitted isotropically from the pulsar magnetosphere, within the last closed magnetic field lines. Observations with time-resolved UV spectroscopy are needed to verify this hypothesis by studying the pulsar spectrum as a function of the rotation phase. In this way, it would be possible to determine whether the spectrum of the DC component differs from that of the pulsed component, and more importantly whether it varies with the rotation phase. This would help to confirm that the source of the DC component is isotropic emission from the pulsar magnetosphere, as we speculated above.

\section{Discussion}

\subsection{The Pulsar UVOIR Spectrum}

We corrected the observed NUV and FUV magnitudes for the effects of interstellar extinction. As was done done in Mignani et al. (2010b, 2012), we assumed a reddening $E(B-V)=0.2$ (see also discussion in Serafimovich et al. 2004). We assumed the interstellar extinction law of Fitzpatrick (1999), which gives extinction correction terms $A_{\mathrm{NUV}}=1.62$ at $\lambda=2359 \AA$ and $A_{\mathrm{FUV}}=1.56$ at $\lambda=1596 \AA$. We remark that our choice of the interstellar extinction correction was made so as to not bias the comparison with the optical and near-IR fluxes of Mignani et al. (2010b, 2012), which were also corrected using the extinction law of Fitzpatrick (1999). By correcting the observed magnitudes according to the computed $A_{\mathrm{NUV}}$ and $A_{\mathrm{FUV}}$, we obtain extinction-corrected fluxes of $F_{\mathrm{NUV}}=(7.88 \pm 0.14) \mu \mathrm{Jy}$ and $F_{\mathrm{FUV}}=(2.40 \pm 0.23) \mu \mathrm{Jy}$ for the NUV and FUV bands, respectively.

We used these flux values to characterize the PSR B0540 -69 spectrum from the near-IR to the UV. Here we are aware that we are comparing flux measurements taken at different epochs, in particular before and after the large change in $\dot{\nu}$
(Marshall et al. 2015). Since the effects of this event on the pulsar UVOIR flux and spectrum are unknown, such a comparison must be made with due care. Figure 3 shows the UVOIR spectrum of PSR B0540-69 together with the best-fit PL to the optical/near-IR fluxes (Mignani et al. 2012). In all cases, the plotted fluxes have been computed through aperture photometry on the time-integrated images. Therefore, the fluxes are integrated over the pulse phase, which means that they account for both pulsed and unpulsed emission components. As can be seen, the NUV and FUV fluxes are clearly incompatible with the slope of the optical/near-IR PL $\left(\alpha_{\mathrm{O} \text {,nIR }}=0.70 \pm 0.04\right)$, with the former above and the latter below its extrapolation by $\sim 25 \sigma$ and $\sim 5.5 \sigma$, respectively, suggesting a drastic turnover in the spectrum at wavelengths below $2400 \AA$. In particular, the NUV and FUV fluxes are not consistent with a flattening of the optical/near-IR PL, as one would expect from the comparison between the optical and X-ray spectra (see Figure 3 of Mignani et al. 2010b), but instead show a steeper PL in the UV, with spectral index $\alpha_{\mathrm{UV}}=3.05 \pm 0.25$. Such a steep PL slope has never been seen in the UVOIR spectra of any other pulsar, where the spectral index is usually $\approx 0-1$ (Mignani 2011). Since this result is unexpected, we double-checked for possible bugs in our end-to-end procedure as follows.

First, we checked that our photometry is affected neither by systematics, such as the aperture correction, nor by calibration issues, such as the zero-point definition, for which we straightforwardly applied values reported in the instrument handbook and in the image headers. As a safe measure, we verified that the tabulated aperture correction factors are consistent with those measured directly on the image and that the values of the PHOTFLAM keywords in the image headers were consistent with those reported in other sources (e.g., Proffit 2006). Therefore, we are confident that the observed magnitudes have been computed and calibrated correctly. We note that most of the fluxes plotted in Figure 3 have been measured with the $H S T$ and calibrated in a similar fashion, which minimizes the risk of cross-calibration problems.

As a test, we computed the pulsar photometry using methods different from those described in Section 3.1, e.g., by employing different software tools for the photometry, by using different apertures and background areas, and consequently different values for the aperture correction, and obtained fully compatible magnitude values, which confirms that our results are robust and method-independent. For consistency, we also compared our measured pulsar CRs in the NUV and FUV images with those predicted by the STIS Exposure Time Calculator (ETC). We assumed the dereddened NUV and FUV fluxes at the peak wavelength of the F25QTZ filter, the PL connecting these two values (Figure 3) as a template spectrum, an $E(B-V)=0.2$, and the zodiacal light at the PSR B0540-69 coordinates. The ETC predicts CRs of 1.48 (NUV) and 0.053 (FUV), after accounting for the sky background from the PWN, which are fully consistent with ours.

In principle, our photometry might have been affected by issues other than those just discussed, such as a glitch in the instrument performances. However, no variations in the detector throughput or other anomalies have been reported in the STIS instrument science reports ${ }^{25}$ for the time frame around

\footnotetext{
25 http://www.stsci.edu/hst/stis/documents/isrs
} 


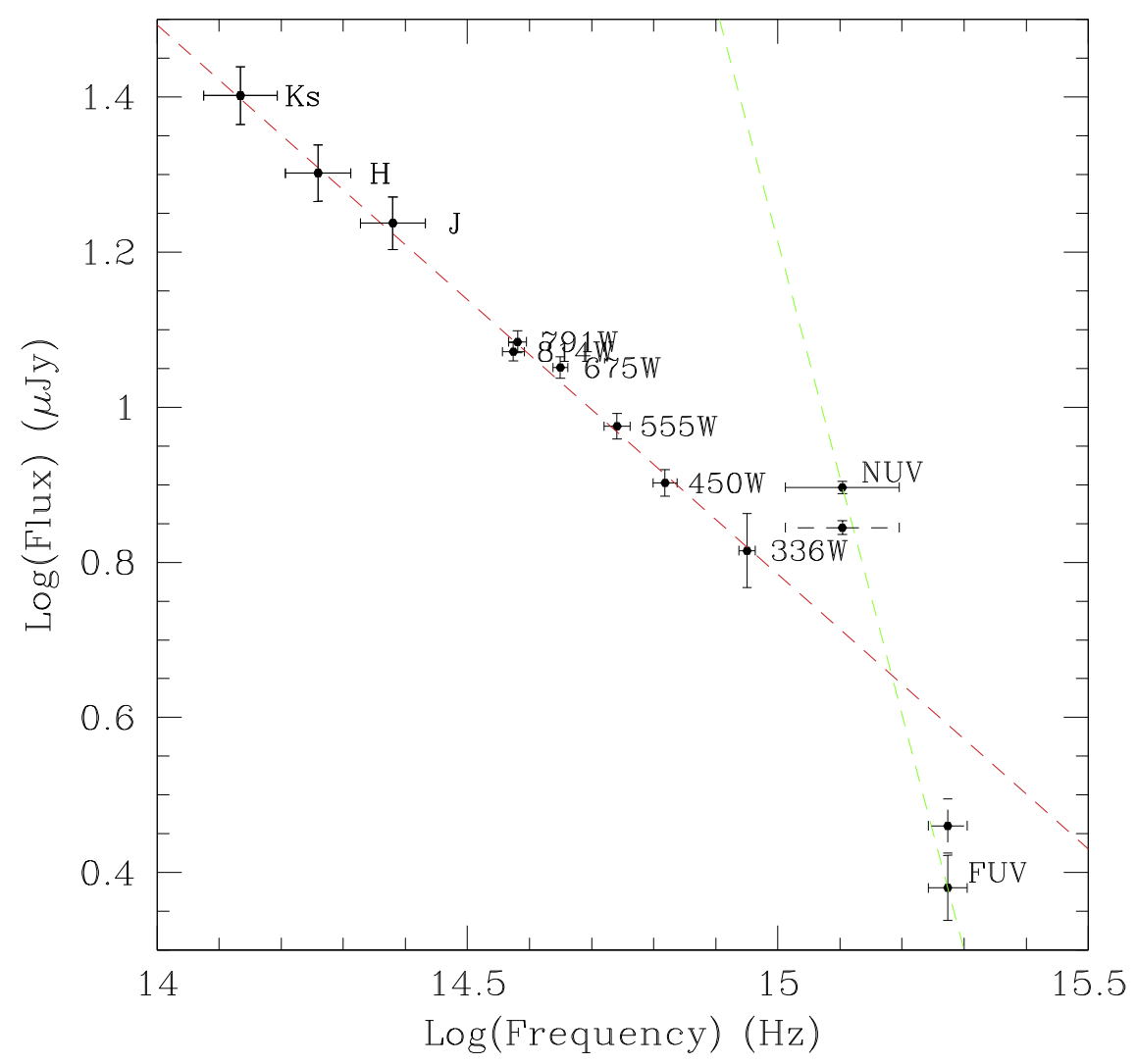

Figure 3. Multi-band spectrum of PSR B0540-69. The HST optical flux measurements, labeled with the broadband (W) filter numbers, are taken from Mignani et al. (2010b), and the VLT near-IR ones $\left(J, H, K_{\mathrm{s}}\right)$ from Mignani et al. (2012). The NUV and FUV fluxes are from the present work. All fluxes have been obtained through imaging photometry and are integrated over the pulse phase. The red line corresponds to the best-fit optical/near-IR PL spectrum computed by Mignani et al. (2012), whereas the green line indicates the PL connecting the NUV and FUV fluxes. Correction for interstellar reddening has been applied as described in Section 4.1, using the extinction law of Fitzpatrick (1999). For comparison, we also plotted the NUV and FUV fluxes (dashed lines) corrected using the extinction law for the 30 Doradus region (Gordon et al. 2003). Applying this law to the optical and near-IR fluxes would not result in an appreciable difference.

our observations. To rule out that the large difference between the pulsar NUV and FUV CRs is due to instrumental effects of some sort we computed the difference between the co-aligned NUV and FUV images and found that the CR residuals for all stars in the field of view are randomly distributed above and below those for the pulsar, as one would expect if the data are free of instrument systematics.

Finally, we have carefully checked the applied UV extinction correction against those derived in more recent works and ruled out that the dereddened NUV and FUV flux values that we derived are substantially mis-estimated. For instance, assuming the extinction law of Gordon et al. (2003), which is derived both for the LMC as a whole and, more specifically, also for the 30 Doradus region, would only marginally change the extinction correction in the NUV and FUV, whereas the extinction correction in the near-IR and the optical bands would be indistinguishable from that derived from the extinction law of Fitzpatrick (1999). Although this would result in a $\approx 12 \%$ lower dereddened NUV flux, and in a similarly higher dereddened FUV flux, it would only partially account for the NUV/FUV flux difference with respect to the optical/near-IR PL extrapolation, which would still be at $\sim 20 \sigma$ and $\sim 3.5 \sigma$, respectively (Figure 3). Since PSR B0540-69 is embedded in its supernova remnant (SNR), one can speculate about a difference in the interstellar extinction law on a more local scale, owing to a different chemical composition of the remnant with respect to the surrounding environment. This speculation, however, cannot be easily verified with the available data, especially given the small angular extent of the remnant $\left(4^{\prime \prime} \times 4^{\prime \prime}\right)$. Even so, since PSR B0540-69 lies within the 30 Doradus nebula $\left(40^{\prime} \times 25^{\prime}\right)$ its effects on the interstellar extinction law dominate over those produced by fluctuations in the local environment.

Having done all the due checks, and having found no obvious bug in our procedure, we are bound to conclude that the source of the large difference between the pulsar NUV and FUV fluxes is intrinsic to the pulsar. This suggests that, unexpected as it may be, the abrupt turnover observed in the pulsar PL spectrum is intrinsic to the source, although the evidence must be supported by more flux measurements in the UV.

Thinking of a physical origin, one may speculate whether the larger (smaller) NUV (FUV) flux with respect to the optical/ near-IR PL extrapolation might be (at least partially) explained by a DC component in the pulsar emission that is stronger in the NUV than at longer wavelengths, whereas it is almost absent from the FUV (see Section 3.2). If the PF were similar at all wavelengths, such a different DC component fraction in the UV would raise the phase-integrated NUV flux above the expected value, and decrease the FUV flux, affecting the slope of the phase-integrated spectrum. Unfortunately, there are no published light curves of PSR B0540-69 obtained in bands other than $V$ (see Gradari et al. 2011 and references therein ${ }^{26}$ ),

\footnotetext{
${ }^{26}$ Middleditch et al. (1987) indeed obtained light curves of PSR B0540-69 in the $U B V R I$ bands but these were never published.
} 
so we do not know the value of the PF at different wavelengths. Moreover, these light curves were all obtained with nonimaging photon counting detectors, making it more problematic to subtract the PWN background and disentangle a genuine DC component in the pulsar emission. Therefore, we can compare neither the pulsed nor the DC component fraction at different wavelengths. Future multi-band observations of PSR B0540-69 at high time and high spatial resolution with imaging photometers would be crucial to test our hypothesis.

Another, but less likely, possibility is that of long-term variability in the pulsar UV flux since the epochs of the optical (2007 June-November) and near-IR (2010 October-December) observations of Mignani et al. (2010b, 2012). Pulsars are generally known to be stable sources on long timescales, and in the case of the Crab it has been shown that optical flux variations can be at the level of just a few millimagnitudes per year (Sandberg \& Sollerman 2009). Larger variations, such as those observed in $\gamma$-rays for PSR J2021+4026 (Allafort et al. 2013), cannot be ruled out a priori. However, in this case one would expect the NUV and FUV fluxes, as well as the optical and near-IR fluxes, to vary in the same direction, unless the variation in flux is accompanied by a variation in spectrum. Unfortunately, there are no multi-epoch sets of UVOIR flux measurements of PSR B0540-69 in which to look for possible flux/spectral variations. Therefore, obtaining a new set of UVOIR flux measurements as close in time as possible to one another is the step required to search for possible long-term variability at these wavelengths. In the X-rays, no significant long-term variability had been observed from the analysis of Rossi X-ray Timing Explorer (RXTE) observations of PSR B0540-69 (Ferdman et al. 2015), which cover a time span of $15.8 \mathrm{yr}$. However, these observations only extend up to 2011 December 3, i.e., right before the large change in $\dot{\nu}$, which occurred sometime between 2011 December 3 and 17 (Marshall et al. 2015).

As anticipated earlier in this section, we cannot rule out that an erratic phenomenon such as the large change in $\dot{\nu}$ might have produced a variation in the pulsar UVOIR flux and/or spectrum, making the UV fluxes not directly comparable with the optical/ near-IR fluxes, which have been measured before 2011. Since there are neither UV flux measurements taken before the change in $\dot{\nu}$ nor new optical/near-IR flux measurements taken after this event to compare with, we cannot establish whether a consequent variation in the pulsar UVOIR flux and/or spectrum ever occurred. The same new set of UVOIR flux measurements required to look for long-term variability (see above) will also help to determine whether the change in $\dot{\nu}$ has affected the pulsar emission at these wavelengths. Interestingly, the last two RXTE observations of PSR B0540-69, on 2011 December 17 and 31, i.e., after the large change in $\dot{\nu}$, do not show any significant difference in the X-ray flux with respect to the historical trend (Marshall et al. 2015). However, no other information on the evolution of the X-ray flux after the event has ever been reported. Therefore, we looked for X-ray variability in a more recent time frame. As a first-order test, we compared the pulsar X-ray flux measured at two epochs closest in time to our near-IR (2010 October-December) and UV (2017 February) observations using data available in X-ray observatory archives. To this aim, the only suitable X-ray data are those in the Swift/XRT archive, taken on 2010 November 10 (13.5 ks) and 2017 February 14 (1.1 ks) in PHOTON and WT modes, respectively. However, from the measured X-ray flux we found no evidence of significant variability between the two epochs. This suggests that the large change in $\dot{\nu}$ did not affect the X-ray flux, as implied by the postevent RXTE observations (Marshall et al. 2015). A systematic analysis of all the Swift/XRT observations of PSR B0540-69 from 2015 February 17 onward (F. E. Marshall et al. 2019, in preparation) will allow us to look for possible variability in X-ray flux over the epoch range of interest in more detail.

The possibility that the excess NUV flux with respect to the optical/near-IR PL extrapolation is due to an emission feature centered at $\sim 2350 \AA$, perhaps associated with an ion cyclotron line produced in the pulsar magnetosphere is, at present, no more than a speculation. The possible presence of emission/absorption features in pulsar optical spectra had been claimed for the Crab (Nasuti et al. 1996), Geminga (Mignani et al. 1998), and PSR B0656+14 (Durant et al. 2011) but the existence of these features either has not been confirmed by independent observations or is still to be proved. An unsubtracted spectral feature in the PSR B0540-69 SNR emitted from a region very close to the pulsar may be another possibility. High-spatial-resolution UV spectroscopy observations of the pulsar and of its SNR are needed to verify these two possibilities. So far, the only optical/UV spectrum of PSR B0540-69 was obtained by Hill et al. (1997) with the Faint Object Spectrograph aboard HST, but the spectral coverage (2500-5000 $\AA$ ) did not extend to the wavelength range of interest.

\subsection{The Pulsar Multi-wavelength Spectrum}

Regardless of the unusual PL slope in the UV, it is clear that the NUV and FUV fluxes measured for PSR B0540-69 would be incompatible with an RJ spectrum $\propto \nu^{2}$. This speaks in favor of a non-thermal (synchrotron) origin of the UV emission, as is believed to be the case for the optical and near-IR emission, powered by the pulsar rotational energy. Under the hypothesis that the change in pulsar $\dot{\nu}$ did not introduce a flux/spectrum variation (see discussion in Section 4.1), the difference in the PL slope from the optical/near-IR to the UV would then imply a break in the pulsar non-thermal UVOIR spectrum.

Breaks in the pulsar non-thermal UVOIR spectra are not unheard of. Indeed, a spectral break is observed in the Crab pulsar in the transition from the optical/near-IR to the UV, where the PL spectral index features a turnover from $-0.31 \pm 0.02$ to 0.11 (Sollerman 2003). This break, however, is clearly not as dramatic as that observed in PSR B0540-69. The Vela pulsar, on the other hand, features a single PL that fits the spectrum all the way from the near-IR to the UV (Zyuzin et al. 2013). Whether the presence or absence of breaks in the pulsar UVOIR spectra depends on the characteristic age, with Vela being a factor of 10 older than the Crab, or on other pulsar parameters is not clear yet (see, e.g., Mignani et al. 2016a). In the case of PSR B0656+14 and Geminga-the other two pulsars that had been detected in the UVOIR - the spectral break between the optical and UV is only due to the onset of the RJ component, which dominates over the PL one in the UV (e.g., Kargaltsev \& Pavlov 2007), and not to a genuine turnover in the optical PL spectral index.

The characterization of the overall pulsar spectral energy distribution (see Figure 3 in Fermi LAT Collaboration et al. 2015) is not significantly advanced by our new NUV and FUV fluxes, given their limited spectral coverage. However, they confirm that the optical/near-IR and X-ray spectra cannot be described by a single PL, as pointed out by Mignani et al. (2010b) and Serafimovich et al. (2004) based on the optical fluxes only. Observations at shorter UV wavelengths would 
Table 1

Pulsars Detected in the UV by the $H S T$

\begin{tabular}{|c|c|c|c|c|c|c|c|c|}
\hline Name & $\begin{array}{c}P_{\mathrm{s}} \\
(\mathrm{ms})\end{array}$ & $\begin{array}{c}\tau_{\mathrm{C}} \\
(\mathrm{kyr})\end{array}$ & $\begin{array}{c}B_{\mathrm{s}} \\
\left(10^{12} \mathrm{G}\right)\end{array}$ & $\left(10^{38} \begin{array}{l}\dot{E} \\
\left.\mathrm{erg} \mathrm{s}^{-1}\right)\end{array}\right.$ & $\begin{array}{c}L_{\mathrm{UV}} \\
\left(10^{34} \mathrm{erg} \mathrm{s}^{-1}\right)\end{array}$ & $\begin{array}{c}d \\
(\mathrm{kpc})\end{array}$ & $\begin{array}{c}\lambda_{\min }-\lambda_{\max } \\
(\AA)\end{array}$ & Instrument \\
\hline Crab (1) & 33.39 & 1.26 & 3.79 & 4.50 & 1.04 & 2 & $1600-3200$ & STIS/NUV-MAMA G230L \\
\hline Vela (3) & 89.32 & 11.3 & 3.38 & 0.069 & $1.1 \times 10^{-4}$ & 0.287 & $1800-3000$ & STIS/NUV-MAMA F25SRF2 \\
\hline B0656+14 (4) & 384.89 & 111 & 4.66 & $3.8 \times 10^{-4}$ & $4.2 \times 10^{-5}$ & 0.288 & $1150-1700$ & STIS/FUV-MAMA G140L \\
\hline Geminga (5) & 237.09 & 342 & 1.63 & $3.2 \times 10^{-4}$ & $1.1 \times 10^{-5}$ & 0.200 & $1800-3000$ & STIS/NUV-MAMA F25SRF2 \\
\hline $\mathrm{B} 1055-52(6)$ & 197.10 & 535 & 1.09 & $3.0 \times 10^{-4}$ & $2.3 \times 10^{-5}$ & 0.35 & $1350-2000$ & ACS/SBC F140LP \\
\hline B0950+08 (8) & 253.06 & $1.75 \times 10^{4}$ & 0.24 & $5.6 \times 10^{-6}$ & $4.3 \times 10^{-6}$ & 0.262 & $1250-2000$ & ACS/SBC F125LP \\
\hline $\mathbf{J} 2124-3358(9)$ & 4.93 & $3.8 \times 10^{6}$ & $3.2 \times 10^{-4}$ & $6.8 \times 10^{-5}$ & $5.8 \times 10^{-6}$ & 0.410 & $1250-2000$ & ACS/SBC F125LP \\
\hline J0437-4715 (10) & 5.96 & $4.9 \times 10^{6}$ & $5.8 \times 10^{-4}$ & $3.8 \times 10^{-5}$ & $4.7 \times 10^{-7}$ & 0.139 & $1150-1700$ & STIS/FUV-MAMA G140L \\
\hline
\end{tabular}

Note. The table lists the values of spin period $P_{\mathrm{s}}$, characteristic age $\tau_{\mathrm{C}}$, surface magnetic field $B_{\mathrm{s}}$, rotational energy loss $\dot{E}$, as listed in the Australia Telescope National Facility (ATNF) pulsar catalog (Manchester et al. 2005), and the UV luminosity $L_{\mathrm{UV}}$ computed from the observed flux for the assumed distance $(d)$ and wavelength range $\left(\lambda_{\min }-\lambda_{\max }\right)$. The last column reports the corresponding instrument/detector combination, either the STIS/MAMAs or the Advanced Camera for Surveys (ACS) Solar Blind Channel (SBC), and the names of the imaging filters (F) or spectroscopic gratings (G) used. For the binary millisecond pulsar PSR J0437-4715 the UV luminosity value refers to the pulsar only. (1) Sollerman et al. (2000); (2) this work; (3) Romani et al. (2005); (4) Shibanov et al. (2005); (5) Kargaltsev et al. (2005); (6) Mignani et al. (2010a); (7) Mignani et al. (2002); (8) Pavlov et al. (2017); (9) Rangelov et al. (2017); (10) Kargaltsev et al. (2004).

help to bridge the pulsar emission in these two spectral regions. Unfortunately, $H S T$ observations cannot push the wavelength limit any further than $\approx 1100 \AA$, whereas PSR B0540-69 would not have been spatially resolved by the imaging detectors aboard the Extreme Ultraviolet Explorer (Bowyer \& Malina 1991) and has not been observed by the Far Ultraviolet Spectroscopic Explorer (Moos et al. 2000). A more robust characterization of the pulsar spectrum in the UV through HST spectroscopy, though, would help to make its extrapolation toward higher frequencies more accurate.

\subsection{The Pulsar UV Luminosity}

PSR B0540-69 is the tenth pulsar detected in the UV by the HST (see Table 1 for a summary). The list includes the recycled millisecond pulsar PSR J0437-4715, which is in a binary system and was spectroscopically resolved from its white dwarf companion (Kargaltsev et al. 2004; Durant et al. 2012). We note that the double pulsar system PSR J0737-3039A/B was also detected in the UV (Durant et al. 2014) but it was not possible to disentangle the contribution of the two pulsars in the time-integrated HST images. For this reason, neither of the two pulsars is included in Table 1.

We computed the UV luminosity of PSR B0540-69 and compared it with that of other pulsars detected in the UV. For PSR B0540-69, the isotropic luminosity in the NUV F25QTZ filter (Riley 2017) is $L_{\mathrm{NUV}}=1.27 \times 10^{34} d d_{\mathrm{LMC}}^{2} \mathrm{erg} \mathrm{s}^{-1}$, where $d_{\mathrm{LMC}}$ is the LMC distance in units of $48.97 \mathrm{kpc}$ (Storm et al. $2011)$. This corresponds to a fraction of $\sim 8.5 \times 10^{-5}$ of its rotational energy loss $\dot{E}\left(1.5 \times 10^{38} \mathrm{erg} \mathrm{s}^{-1}\right)$. In the FUV F25QTZ filter, the luminosity is $L_{\mathrm{FUV}}=1.9 \times 10^{33} d_{\mathrm{LMC}}^{2}$ $\mathrm{erg} \mathrm{s}^{-1}$ and the $\dot{E}$ fraction is correspondingly lower by a factor $\approx 6.6$. For comparison, in the near-IR ( $K$ band) this fraction is $\sim 1.8 \times 10^{-5} d_{\text {LMC }}^{2}$ (Mignani et al. 2012), whereas in the optical ( $V$ band) it is $\sim 1.7 \times 10^{-5} d_{\text {LMC }}^{2}$ (Mignani et al. 2010b), which means that PSR B0540-69 radiates a factor of five more energy in the NUV than at longer wavelengths.

For the other two young pulsars, Crab and Vela, the UV emission is also non-thermal. For the Crab, integrating its STIS/ NUV-MAMA spectrum (Sollerman et al. 2000) over the NUV
F25QTZ wavelength range gives $L_{\mathrm{NUV}}=1.04 \times 10^{34} \mathrm{erg} \mathrm{s}^{-1}$ for a distance of $2 \mathrm{kpc}$ (Manchester et al. 2005), about the same luminosity as PSR B0540-69. In the case of the Crab, however, owing to its three times larger $\dot{E}$ with respect to PSR B0540-69, this corresponds to an $\dot{E}$ fraction of only $\sim 2.28 \times 10^{-5}$. For Vela, the UV luminosity, obtained from STIS/NUV-MAMA images but in the F25SRF2 filter (Romani et al. 2005), which is similar to the F25QTZ one, is $L_{\mathrm{NUV}}=1.1 \times 10^{29} \mathrm{erg} \mathrm{s}^{-1}$ for the radio parallactic distance of $287 \mathrm{pc}$ (Dodson et al. 2003). This corresponds to a fraction as low as $\sim 1.55 \times 10^{-8}$ of its $\dot{E}(6.9 \times$ $10^{36} \mathrm{erg} \mathrm{s}^{-1}$ ), a factor of 10 higher than the corresponding $\dot{E}$ fraction emitted in the optical, though $\left(\sim 1.95 \times 10^{-9}\right.$; Moran et al. 2013). This shows that also in the UV, as in the optical, Vela emits a lower fraction of its $\dot{E}$ than the very young pulsars Crab and PSR B0540-69.

Strictly speaking, a direct comparison with the UV luminosity of the middle-aged pulsars PSR B0656+14, Geminga, and PSR B1055-52 (Kargaltsev et al. 2005; Shibanov et al. 2005; Mignani et al. 2010a) would not be very informative because of the difference in the underlying emission mechanisms. For the middleaged pulsars the UV emission is dominated by thermal radiation from the cooling neutron star surface and not by non-thermal radiation from the neutron star magnetosphere, as in the case of the young Crab, Vela, and PSR B0540-69. The UV emission is also thermal for the $\sim 17.5 \mathrm{Myr}$ old PSR B0950+08 (Pavlov et al. 2017) and the $\sim 4.9$ Gyr old recycled millisecond pulsar PSR J0437-4715 (Kargaltsev et al. 2004), whereas for both the 2 Myr old PSR B1929+10 (Mignani et al. 2002) and the $\sim 3.8$ Gyr old recycled millisecond pulsar PSR J2124-3358 (Rangelov et al. 2017) the available spectral information is not sufficient to determine whether the UV emission is thermal or nonthermal. As a further complication, in many cases the UV flux values reported in the literature have been obtained with different HST instruments, different techniques (imaging photometry or spectroscopy), and in different wavelength ranges, which makes the inferred UV luminosities not directly comparable to each other.

Therefore, given the very small sample (Crab, Vela, and PSR B0540-69) it is difficult to speculate about possible trends in the pulsar non-thermal UV luminosity as a function of the pulsar parameters, e.g., the surface magnetic field $B_{\mathrm{S}}$ or the rotational 


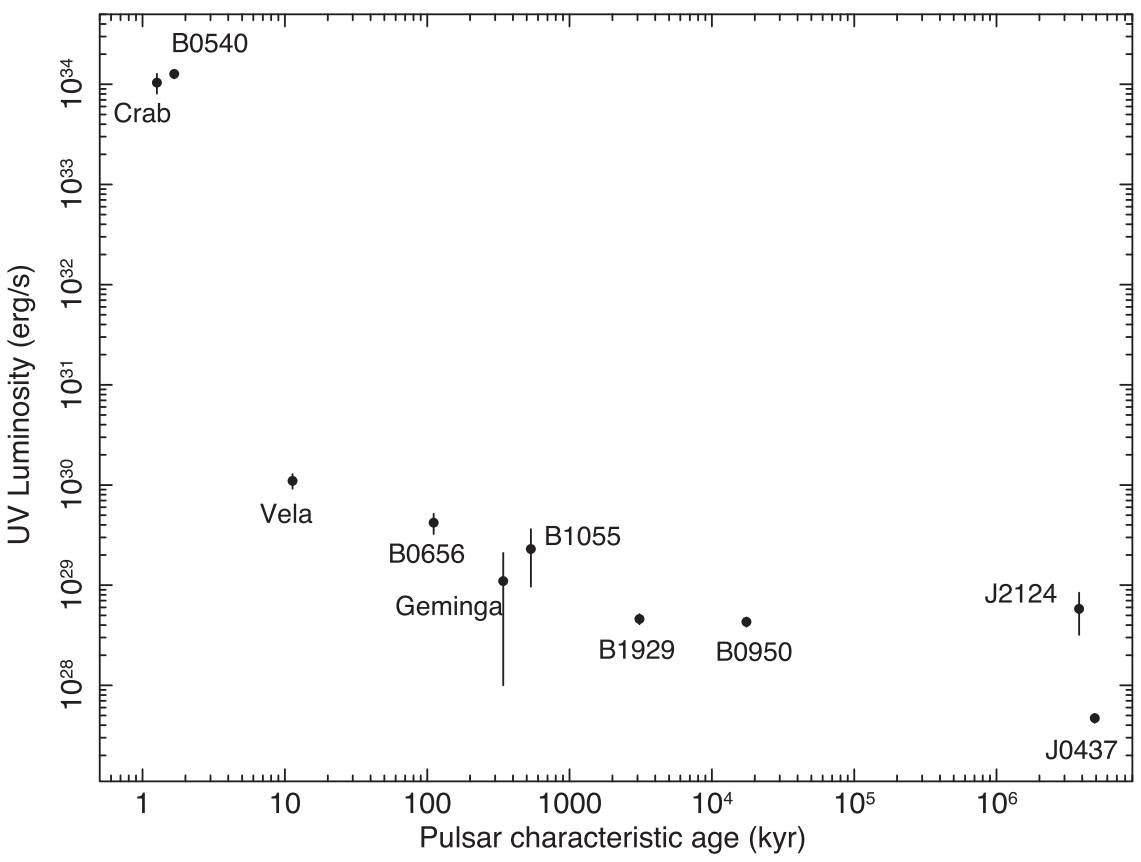

Figure 4. UV luminosity plotted as a function of characteristic age for all pulsars listed in Table 1. Uncertainties in luminosity account for errors in both distance and photometry.

energy loss $\dot{E}$. On the other hand, for the other pulsars the thermal UV luminosity is expected to be insensitive to these parameters, if emitted from a large fraction of the neutron star surface and not from hot polar caps, but to be sensitive to the temperature of the emitting region. Since in the UV we see only the RJ part of the thermal spectrum, the brightness temperature $T_{\mathrm{B}}$ is parameterized by the second power of the ratio between the pulsar distance and the radius of the emitting region, which cannot be easily determined. Indeed, in the lack of modulations at the pulsar spin period in the thermal UV emission the only hard limit is imposed by the radius of the neutron star predicted by different equations of state. This means that deriving a temperature value for comparison with, e.g., models of neutron star cooling comes with significant uncertainties. For the sake of completeness, in Table 1 we reported the luminosity values for all pulsars detected in the UV regardless of the nature of the emission. For illustrative purposes, Figure 4 shows the pulsar UV luminosity $L_{\mathrm{UV}}$ as a function of the characteristic age $\tau_{\mathrm{C}}$ for all pulsars in Table 1. As can be seen, the UV luminosity quickly drops for ages above $\sim 10 \mathrm{kyr}$, i.e., about that of the Vela pulsar, and the trend more or less flattens above $\sim 100 \mathrm{kyr}$. This is expected since the contribution of the UV non-thermal emission becomes less important for pulsars older than $\sim 100 \mathrm{kyr}$. A similar trend has been found for the pulsar optical luminosity (e.g., Zharikov \& Mignani 2013), marking also in this case the difference between young and middle-aged/old pulsars, although for the latter the contribution of the non-thermal emission can still be important.

\subsection{The Pulsar UV and Optical Light Curves}

Being close in wavelength, it is natural to compare first the UV light curves of PSR B0540-69 to those in the optical band. $^{27}$ In the optical, its most recent light curve has been published by Gradari et al. (2011) based on data obtained with the Iqueye instrument (Naletto et al. 2009) at the ESO New

\footnotetext{
27 PSR B0540-69 has been detected in the near-IR (Mignani et al. 2012), but pulsations in this band have not yet been measured.
}

Technology Telescope (NTT) in 2009 January and December. Iqueye observations taken during the same observing runs were also used to produce an updated optical light curve of the Vela pulsar (Spolon et al. 2019). The light curve profile of PSR B0540-69 clearly revealed a two-peak structure, with the two peaks separated in phase by $\sim 0.3$, in agreement with all the optical light curves of PSR B0540-69 reported in the literature (see Gradari et al. 2011 and references therein). Figure 5 (top) shows the light curve built from the Iqueye data of Gradari et al. (2011), as published in Figure 2 of Fermi LAT Collaboration et al. (2015).

We note that Gradari et al. (2011) found possible evidence (at the $\approx 3 \sigma$ level) of a third peak in the light curve interposed between the two main peaks (see their Figure 1) but this is not visible in Figure 5 (top). The reason behind this discrepancy, never addressed so far, is that the Iqueye data have been fully reprocessed by Fermi LAT Collaboration et al. (2015) with an upgraded version of the data reduction software, which improved the determination of the photon time of arrival. Another, and likely more important, reason is the use of a different ephemeris for folding the light curve. Gradari et al. (2011) did not have simultaneous ephemeris available and then folded and aligned the data on the basis of their own period measurements, whereas Fermi LAT Collaboration et al. (2015) used the ephemeris derived from observations with the $R X T E$ Proportional Counter Array (PCA), between 2008 May 16 and 2011 December 3 (MJD 54602-55898). Therefore, the third peak seen in the Iqueye data by Gradari et al. (2011) was probably an artifact of the data analysis. Interestingly, this peak might correspond to that possibly seen between the two main peaks in the NUV light curve (Figure 2, top), whose significance, however, is also marginal (see Section 3.2). Although two coincidences may represent a clue, only followup optical/UV observations, possibly with different telescope/ instrumental setups, can provide more convincing evidence of the existence of this putative third peak. Confirming its existence would unveil a more complex light curve 


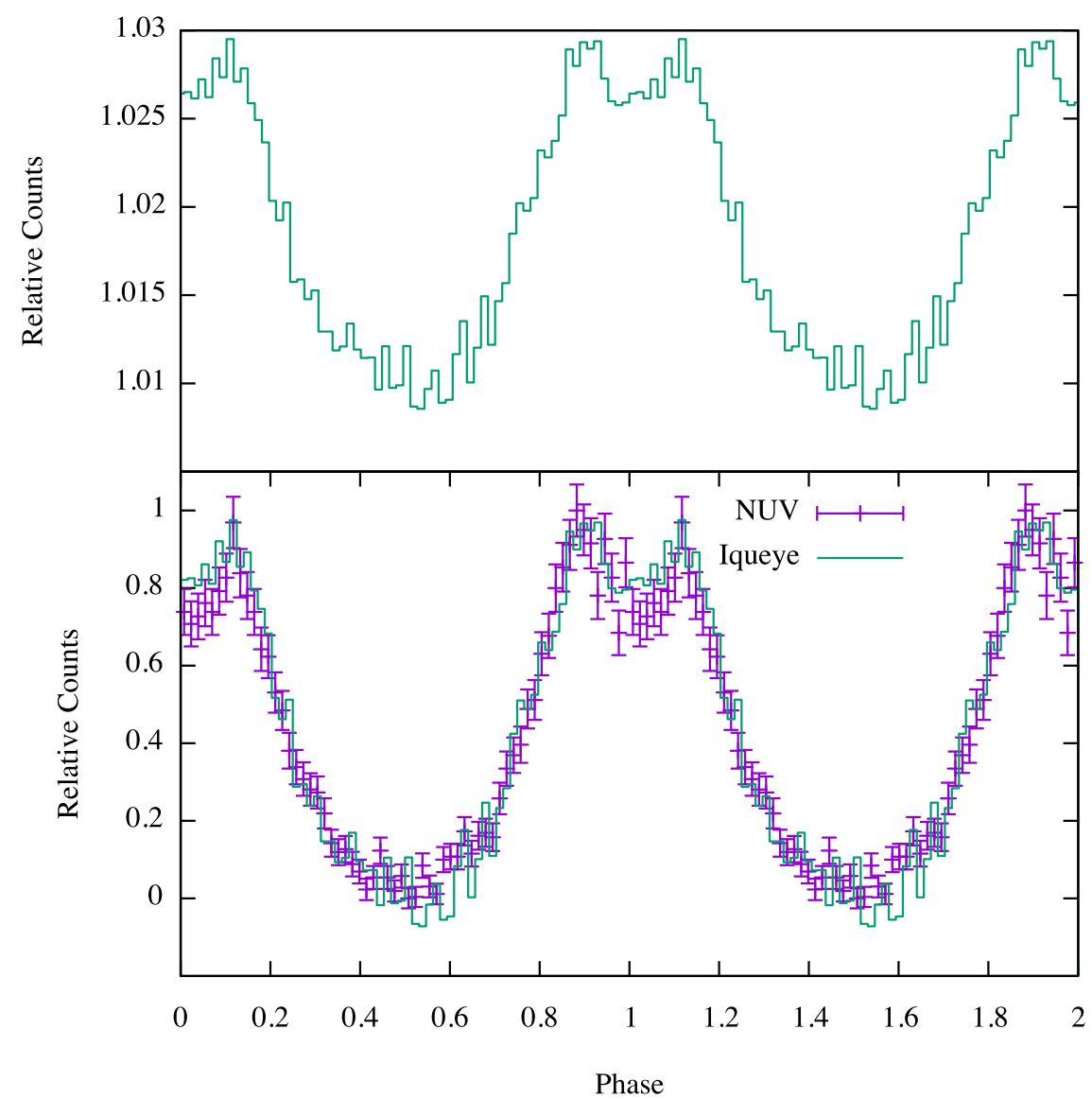

Figure 5. Top: optical light curve taken through the white band filter reconstructed from a reanalysis of the Iqueye data of Gradari et al. (2011). Two cycles are shown for clarity. Since these data were obtained back in 2009, i.e., before the large change in the pulsar $\dot{\nu}$ (Marshall et al. 2015), the light curve has been folded using the ephemeris derived from RXTE/PCA observations over the period 2008 May 16-2011 December 3 (Fermi LAT Collaboration et al. 2015). The third peak tentatively seen in the Iqueye light curve published in Gradari et al. (2011) is not visible here; see Section 4.4 for details. Bottom: NUV light curve (magenta line) from Figure 2 superimposed on that constructed from the Iqueye data set (green line).

morphology than initially thought, which might encode thus far missing information on the pulsar viewing and beaming angles and the structure of the optical/UV emission cone.

As can be seen from the comparison between Figure 2 and Figure 5 (top), the NUV/FUV light curve profiles resemble the optical one, with two peaks separated in phase by approximately the same amount. This resemblance is more noticeable for the NUV light curve, as shown by a direct comparison in Figure 5 (bottom), where the two peaks have similar relative intensities, as in the optical light curve. This means that the difference in the pulsar PL spectrum between the optical and the UV (Section 4.1) did not affect the light curve profile. The UV and optical light curves are also aligned in phase, although they correspond to different epochs (2017 and 2009) and have been folded using different sets of ephemerides owing to the large change in the pulsar $\dot{\nu}$ that occurred between 2011 December 3 and 17 (Marshall et al. 2015). The UV light curves have been folded using the Swift/XRT ephemeris of 2015 February 17-2018 March 28 (F. E. Marshall et al. 2019, in preparation), as explained in Section 3.2, whereas the optical light curve has been folded using the RXTE/PCA ephemeris of 2008 May 16-2011 December 3 (Fermi LAT Collaboration et al. 2015), as explained above. A question then arises about whether the observed alignment is real or whether the large change in $\dot{\nu}$ of 2011 December might have introduced a systematic phase offset, so that the 2017 (UV) and 2009 (optical) light curves of PSR B0540-69 would not be directly comparable to each other. This is a key point for our analysis, aimed at determining whether the observed break between the optical/near-IR and UV PL spectra has consequences not only for the profile of the optical and UV light curves but also for their alignment in phase. Since there are no UV (optical) light curves of PSR B0540-69 obtained before (after) 2011 December 3 for a direct comparison we cannot directly clarify this point. In X-rays and $\gamma$-rays, however, the comparison between light curves obtained before and after the large change in $\dot{\nu}$ does not show any obvious misalignment (see Section 4.5). This suggests that this event did not introduce a major phase offset, at least at high energies, and we can reasonably assume that this is also the case for the optical and UV, although our hypothesis can only be confirmed by new optical timing observations for comparison with those of Gradari et al. (2011).

Therefore, the difference in slope of the pulsar PL spectrum between the optical/near-IR and the UV, if intrinsic to the UVOIR spectrum and not ascribed to spectral/flux variability (see discussion in Section 4.1), would have no consequences for the phase alignment of the optical and UV light curves. Their close resemblance (Figure 5, bottom) independently supports the evidence based on the spectrum that, as in the optical, the UV radiation is of magnetospheric origin. In particular, it suggests that the optical radiation and UV radiation have very similar emission geometries, whereas the 
almost perfect phase alignment between the peaks suggests that the emission region in these two bands is most likely the same.

\subsection{The Pulsar Multi-epoch Light Curves}

As anticipated in the previous section, here we describe the results of the comparison between the X-ray and $\gamma$-ray light curves of PSR B0540-69 obtained at different epochs, carried out in this work for the first time.

Figure 6 shows two sets of X-ray and $\gamma$-ray light curves of PSR B0540-69. The first set (panels (A) and (C)) corresponds to an epoch range antecedent to the beginning of 2012 (hereafter "pre-2012"), i.e., before the large change in $\dot{\nu}$ that occurred between 2011 December 3 and 17 (Marshall et al. 2015). In particular, Figure 6(A) shows the RXTE/PCA X-ray light curve built by integrating all data taken between 2008 May 16 and 2011 December 3 (MJD 54602-55898), whereas Figure 6(C) shows the Fermi/LAT $\gamma$-ray light curve built from contemporary data taken between 2008 August 5 and 2011 December 3 (MJD 54682-55898). Both the RXTE/PCA and Fermi/LAT data are the same as used in Fermi LAT Collaboration et al. (2015). In both panels, the light curves have been folded using the pre-2012 ephemeris obtained from the full (MJD 54602-55898) RXTE/PCA data set, as done in Fermi LAT Collaboration et al. (2015). The second set (panels (B) and (D)) corresponds to an epoch range subsequent to the end of 2014 (hereafter "post-2014"), i.e., after the large change in $\dot{\nu}$. The X-ray and $\gamma$-ray light curves are built by integrating all data taken with the Swift/XRT between 2015 February 17 and 2018 March 28 (MJD 57070-58205) and with the Fermi/ LAT between 2015 February 17 and 2018 June 1 (MJD 57070-58270), respectively. Therefore, both data sets cover the epoch range around our HST observations. The Swift/XRT data are the same as described in Section 3.2 and have been partially published in Marshall et al. (2015, 2016), whereas the new Fermi/LAT data have not been published before. In both panels, the light curves have been folded using the post-2014 ephemeris obtained from the full (MJD 57070-58205) Swift/ XRT data set (F. E. Marshall et al. 2019, in preparation), which has been used to fold our HST/STIS light curves (Section 3.2).

For consistency, we analyzed both the pre-2012 and post2014 Fermi /LAT data sets, which cover virtually identical time spans $(\approx 1200$ days $)$, using exactly the same procedure. In particular, we produced $\gamma$-ray light curve profiles for PSR B0540-69 by using Pass 8 Source class events, analyzing photons with energies above $0.1 \mathrm{GeV}$ and with reconstructed directions within $8^{\circ}$ of the pulsar. Events with zenith angles above $105^{\circ}$ were rejected, to limit the contamination caused by the Earth's limb. Phase calculations were carried out using the Fermi plugin (Ray et al. 2011) of TEMPO2 (Hobbs et al. 2006). In order to improve the signal-to-noise ratio of the $\gamma$-ray light curve for both the pre-2012 and post-2014 time intervals, we assigned weights to the individual photons using the weighting method described in P. Bruel et al. (2019, in preparation). The weights give the probabilities that the individual photons originated from PSR B0540-69. We find that using $\log _{10} E_{r}=3.2$ where $E_{r}$ is the reference energy in $\mathrm{MeV}$ of the weighting algorithm (see Bruel et al. for a description) optimizes the signal-to-noise ratio of the profiles. The pre-2012 Fermi/LAT light curve profile (Figure $6(\mathrm{C})$ ), built using the weighting method described above, is consistent with that

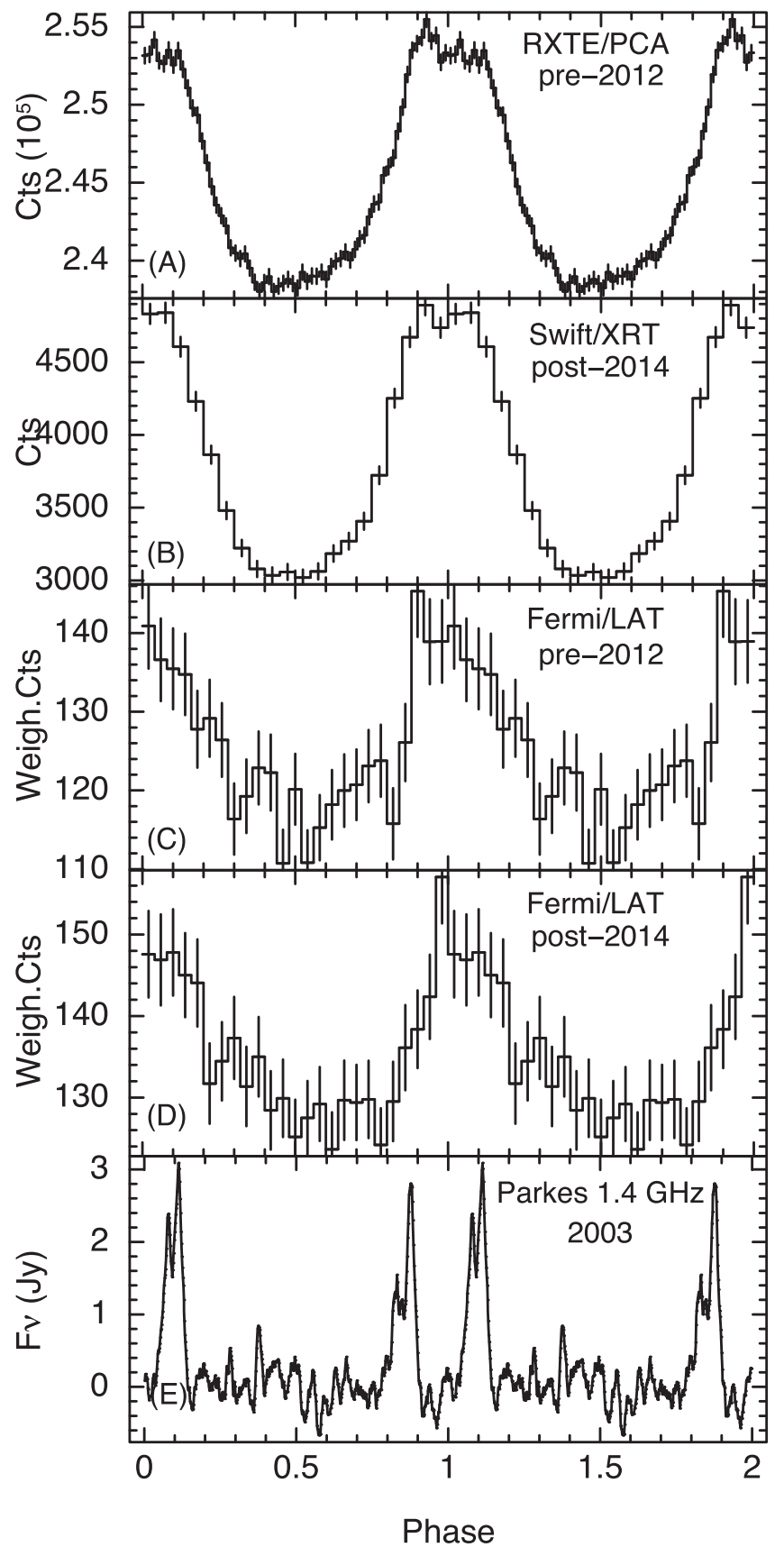

Figure 6. X-ray and $\gamma$-ray light curves of PSR B0540-69 obtained from data taken before (pre-2012) and after (post-2014) the large change in $\dot{\nu}$ that occurred between 2011 December 3 and 17 (Marshall et al. 2015). From top to bottom: RXTE/PCA X-ray light curve (MJD 54602-55898), Swift/XRT X-ray light curve (MJD 57070-58205), Fermi/LAT $\gamma$-ray light curves (MJD 54689-55898 and MJD 57070-58270). Both the pre-2012 and post-2014 LAT light curves have been built using using the photon weighting method described in P. Bruel et al. (2019, in preparation). In all cases, the light curves have been built by integrating all the data collected over the time intervals reported above in parentheses. The pre-2012 RXTE/PCA and Fermi/LAT light curves are based on the same data as used in Fermi LAT Collaboration et al. (2015). In the first and third panels, the light curves have been folded using the pre-2012 ephemeris obtained from the RXTE/PCA data set (Fermi LAT Collaboration et al. 2015), whereas in the second and fourth panels they have been folded using the post-2014 ephemeris obtained from the Swift/XRT observations (F. E. Marshall et al. 2019, in preparation). The bottom panel shows, as a reference, the radio light curve at $1.4 \mathrm{GHz}$ obtained from Parkes in 2003 August (Johnston et al. 2004), which is the same as shown in Fermi LAT Collaboration et al. (2015). 
presented in Fermi LAT Collaboration et al. (2015), indicating that we did not introduce any bias or systematic effect.

As can be seen from the comparison between the RXTE/ PCA and Swift/XRT light curves (Figures 6(A) and (B)), the $\mathrm{X}$-ray light curve profile has not changed appreciably between the two epochs. The light curves are qualitatively similar, both featuring two peaks superimposed on a broad pulse, although the $R X T E /$ PCA light curve benefits from better statistics. Furthermore, the two light curves appear to be essentially aligned in phase. This is also true for the pre-2012 and post2014 Fermi/LAT light curves (Figures 6(C) and (D)). No significant variation is observed between the pre-2012 and post-2014 LAT light curve profiles either, although the former seems to feature a more pronounced emission in the phase interval corresponding to the off-pulse region. This off-pulse emission component was already noticed by Fermi LAT Collaboration et al. (2015), who could not determine whether this was associated with the pulsar or its PWN/SNR or with residual emission from the LMC. A more detailed analysis of the $\gamma$-ray data, which is beyond the goals of this work, is needed to determine how the significance of this excess depends on the modeling and subtraction of the background, on the binning used in the light curve, and perhaps on the count statistics.

Ours is the first high-energy follow-up of PSR B0540-69 after its large change in $\dot{\nu}$ (Marshall et al. 2015). The above comparison shows that this event did not introduce either a major phase offset or profile variation in the pulsar light curves, implying that the emission geometry did not change appreciably between the two epoch ranges explored. Furthermore, the comparison between two pre-2012 and post-2014 Swift/XRT observations (Section 4.1) shows that the change in $\dot{\nu}$ did not introduce a variation in the X-ray flux. A qualitative comparison of the counts in the pre-2012 and post-2014 Fermi/LAT light curves (Figures 6(C) and (D)), which are directly comparable to each other (see above), suggests that no variation has occurred in the $\gamma$-ray flux either. This conclusion will be verified by an in-depth analysis of the two Fermi/LAT data sets, whose results will be published in a follow-up paper. Therefore, based on current evidence, we conclude that the event had no consequence for the high-energy emission properties of the pulsar.

\subsection{The Pulsar Multi-wavelength Light Curves}

Here, we briefly describe the comparison between the optical/UV and the X-ray/ $\gamma$-ray light curves and discuss the implications for our understanding of the pulsar emission geometry.

In general, the UV light curves of PSR B0540-69 (Figure 2) fit very well the picture of a multi-wavelength light curve profile characterized by a broad pulse with two peaks, as emerged from optical (Figure 5) and X-ray (Figures 6(A) and (B)) observations; see Figure 2 of Fermi LAT Collaboration et al. (2015). There is no noticeable shift in the pulse phase across the UV/optical/X-ray light curves and there is no evidence of a variation either in the peak separation or in the relative peak intensity as a function of energy. These two peaks are not apparent in the $\gamma$-ray light curve (Figures 6(C) and (D)) though, possibly because of the lower count statistics and larger errors, whereas the alignment in phase with the UV/optical/ $\mathrm{X}$-ray light curves is maintained. For comparison, Figure 6(E) shows the radio light curve of PSR B0540-69 at $1.4 \mathrm{GHz}$ obtained in 2003 August from the Parkes radio telescope (Johnston et al. 2004), also shown in Fermi LAT Collaboration et al. (2015). Unfortunately, owing to the faintness of the pulsar in radio, it was not possible to obtain more recent observations. Indeed, the radio light curve shown in Figure 6(E), the last to be published, was built exploiting the occurrence of 18 bright giant radio pulses (Johnston et al. 2004). As can be seen, the radio light curve profile, with two distinct narrow and structured peaks, is visually different from those at higher energies, suggesting a different emission geometry. Interestingly, the radio peaks are essentially aligned in phase with those observed in the X-ray, optical, and UV light curves, assuming in this last case no major phase offset in radio after the large change in $\dot{\nu}$ (Marshall et al. 2015).

Such a self-similar and phase-aligned light curve profile across different energy ranges is quite remarkable if compared to that of other young pulsars, such as Vela (Romani et al. 2005). In particular, while the similarity of the light curve profiles suggests a similar emission geometry, their alignment in phase suggests that the pulsed multi-wavelength emission in PSR B0540-69 originates from regions very close to one another in the neutron star magnetosphere. A more or less selfsimilar and phase-aligned light curve profile across the opticalto- $\gamma$-ray energy ranges is also observed in the Crab pulsar. These are the only two pulsars featuring this distinctive characteristic, which strengthens the link between PSR B0540 -69 and its "twin." However, the still-limited number of pulsars seen to pulsate from the optical to $\gamma$-rays (five; Section 3.2) makes it difficult to establish whether such an alignment is the rule or the exception. Detecting multiwavelength pulsations from a larger pulsar sample is obviously needed to address this issue. The middle-aged ( $\tau_{\mathrm{C}} \sim 0.5 \mathrm{Myr}$ ) pulsar PSR B1055-52 ( $\left.P_{\mathrm{s}} \sim 197 \mathrm{~ms}\right)$, detected in radio, optical, UV, X-rays, and $\gamma$-rays but not yet in the near-IR (Mignani et al. 2010a), is the most obvious target to search for UV pulsations ${ }^{28}$ and compare the light curve profile with those already measured in radio, X-rays, and $\gamma$-rays. The other young $\left(\tau_{\mathrm{C}} \sim 4900 \mathrm{yr}\right)$ LMC pulsar PSR J0537-6910 $\left(P_{\mathrm{s}} \sim 16 \mathrm{~ms}\right)$ in the N157B SNR would ideally be the best target owing to an $\dot{E} \sim 4.9 \times 10^{38} \mathrm{erg} \mathrm{s}^{-1}$, the largest in the pulsar family. However, so far it has eluded detections at energies other than in X-rays, where it was discovered as an X-ray pulsar (Marshall et al. 1998), and in $\gamma$-rays, although pulsations have not yet been detected in the latter case (Fermi LAT Collaboration et al. 2015). No radio, optical, or UV counterpart has been found despite multiple attempts (e.g., Crawford et al. 2005; Mignani et al. 2005, 2007).

Searching for near-IR pulsations from PSR B0540-69, never detected in any pulsar other than the Crab (Eikenberry et al. 1997), would be the next goal toward completing the multi-wavelength picture for this source and would allow for a full band-to-band comparison with its "twin." In particular, near-IR observations at high time and spatial resolution would help to disentangle the contribution to the light curve from a possible DC component in the pulsar emission from that of the PWN background. From the comparison with our UV light curves, and with those in the optical band, also to be obtained through observations at high time and spatial resolution, it will

\footnotetext{
28 The pulsar is at $\sim 4^{\prime \prime}$ from a $14.6 \mathrm{mag}$ star and it has been detected in the optical with the HST, which, however, has no instrument for high-timeresolution observations above $3000 \AA$ after the decommissioning of the High Speed Photometer.
} 
then be possible to determine whether the DC component fraction remains constant or evolves with wavelength. As discussed in Section 4.1, a different contribution from the DC component in the UV with respect to the optical/near-IR could help to explain the abrupt UV turnover in the pulsar spectrum.

\section{Summary and Conclusions}

Using the STIS MAMAs aboard HST, we detected the LMC pulsar PSR B0540-69 in two UV bands centered around $2350 \AA$ (NUV) and $1590 \AA$ (FUV) and measured pulsations at the pulsar spin period in both bands. This is the first time that PSR B0540-69 has been detected and seen to pulsate in the UV. Aside from the radio, PSR B0540-69 is now one of the five pulsars (counting the radio-quiet Geminga) detected in five different energy bands (near-IR, optical, UV, X-rays, $\gamma$-rays) and seen to pulsate in at least four of them. PSR B1055-52, detected in all these bands but the near-IR (Mignani et al. 2010a), could be next in the list. We also detected the PSR B0540-69 PWN in our NUV observation, with a morphology similar to that observed in the optical and near-IR, but not in the FUV, which indicates a sharp decrease of the PWN surface brightness at shorter wavelengths.

The UV light curves of PSR B0540-69 feature a prominent broad pulse with two peaks very close in phase, similarly to that observed in the optical and X-rays. A significant DC component is also observed in the NUV light curve, possibly associated with unpulsed isotropic emission from the neutron star magnetosphere. As in the Crab pulsar, the UV light curves are also aligned in phase with those in the radio, optical, $\mathrm{X}$-rays, and $\gamma$-rays, although these are not always contemporary with one another. Thus, it seems that the large change in the spin frequency derivative $\dot{\nu}$ that occurred at the end of 2011 (Marshall et al. 2015) did not introduce a major phase offset, as we demonstrated, at least at high energies, from the qualitative comparison between the RXTE/PCA, Swift/XRT, and Fermi/ LAT light curves of PSR B0540-69 obtained before and after the event. The pulsar UV fluxes clearly deviate from the extrapolation at shorter wavelengths of the best-fit PL to the optical/near-IR fluxes $\left(\alpha_{\mathrm{O}, \mathrm{nIR}} \sim 0.7\right.$; Mignani et al. 2012). Under the hypothesis of no long-term flux variability, this would point to an abrupt steepening of the PL spectrum in the UV $\left(\alpha_{\mathrm{UV}} \sim 3\right)$. This has not yet been observed in other pulsars and its explanation remains a challenge.

More HST observations are necessary to independently confirm the difference in the pulsar PL slope in the UV and obtain a better characterization of the pulsar spectrum at wavelengths below $3000 \AA$, which so far is based on our two flux measurements only. This would require multi-band UV photometry with the Advanced Camera for Surveys or, better yet, UV spectroscopic observations with either the STIS or the Cosmic Origins Spectrograph. In particular, time-resolved UV spectroscopy with the STIS (125 $\mu$ s resolution) would enable one to better decouple the spectrum of the pulsar from that of its PWN and look, for the first time, for possible variations in the pulsar PL spectrum as a function of the rotation phase of the neutron star. This would be important to track possible differences in the properties of the emitting particles (density, velocity) in different regions of the neutron star magnetosphere, which are seen as the neutron star rotates.

We thank the anonymous referee for his/her careful review of our manuscript. R.P.M. acknowledges financial support from an INAF "Occhialini Fellowship" and thanks Ralf Siebenmorgen (ESO) for advice on the interstellar extinction correction. We thank our HST Program Coordinator, Denise Taylor (STScI), for constant support in the observation planning and Tony Sohn (STScI) for checking the STIS data status. We thank Patrizia A. Caraveo for comments and suggestions. B.R. acknowledges financial support from the National Science Centre, Poland Grant 2017/25/B/ST9/ 00385. Based in part on observations made with the ESO NTT telescope at the La Silla Paranal Observatory under programmes 082.D-0382 and 084.D-0328(A). The Fermi LAT Collaboration acknowledges generous ongoing support from a number of agencies and institutes that have supported both the development and the operation of the LAT as well as scientific data analysis. These include the National Aeronautics and Space Administration and the Department of Energy in the United States, the Commissariat à l'Energie Atomique and the Centre National de la Recherche Scientifique/Institut National de Physique Nucléaire et de Physique des Particules in France, the Agenzia Spaziale Italiana and the Istituto Nazionale di Fisica Nucleare in Italy, the Ministry of Education, Culture, Sports, Science and Technology (MEXT), High Energy Accelerator Research Organization (KEK), and Japan Aerospace Exploration Agency (JAXA) in Japan, and the K. A. Wallenberg Foundation, the Swedish Research Council, and the Swedish National Space Board in Sweden. Additional support for science analysis during the operations phase is gratefully acknowledged from the Istituto Nazionale di Astrofisica in Italy and the Centre National d'Études Spatiales in France. This work performed in part under DOE Contract DE-AC02-76SF00515.

Facilities: Hubble Space Telescope, Neil Gehrels Swift Observatory, Fermi Gamma-ray Space Telescope, New Technology Telescope.

\section{ORCID iDs}

R. P. Mignani (ib https://orcid.org/0000-0002-8685-583X

L. Guillemot (iD https://orcid.org/0000-0002-9049-8716

B. Rudak (iD https://orcid.org/0000-0003-0452-3805

L. Zampieri (i) https://orcid.org/0000-0002-6516-1329

\section{References}

Allafort, A., Baldini, L., Ballet, J., et al. 2013, ApJL, 777, L2 Bowyer, S., \& Malina, R. F. 1991, AdSpR, 11, 205

Boyd, P. T., van Citters, G. W., Dolan, J. F., et al. 1995, ApJ, 448, 365

Buccheri, R., Bennett, K., Bignami, G. F., et al. 1983, A\&A, 128, 245

Burrows, D. N., Hill, J. E., Nousek, J. A., et al. 2005, SSRv, 120, 165

Caraveo, P. A., Bignami, G. F., Mereghetti, S., \& Mombelli, M. 1992, ApJL, 395, L103

Cocke, W. J., Disney, M. J., \& Taylor, D. J. 1969, Natur, 221, 525

Crawford, F., McLaughlin, M., Johnston, S., Romani, R., \& Sorrelgreen, E. 2005, AdSpR, 35, 1181

De Luca, A., Mignani, R. P., Caraveo, P. A., \& Bignami, G. F. 2007, ApJL, 667, L77

Dodson, R., Legge, D., Reynolds, J. E., \& Mc Culloch, P. M. 2003, ApJ, 596, 1137

Durant, M., Kargaltsev, O., \& Pavlov, G. G. 2011, ApJ, 743, 38

Durant, M., Kargaltsev, O., Pavlov, G. G., et al. 2012, ApJ, 746, 6

Durant, M., Kargaltsev, O., \& Pavlov, G. G. 2014, ApJL, 783, L22

Eikenberry, S. S., Fazio, G. G., Ransom, S. M., et al. 1997, ApJ, 477, 465

Ferdman, R. D., Archibald, R. F., \& Kaspi, V. M. 2015, ApJ, 812, 95

Fermi LAT Collaboration, Ackermann, M., Albert, A., et al. 2015, Sci, 350,801

Fitzpatrick, E. L. 1999, PASP, 111, 63

Gold, T. 1968, Natur, 218, 731 
Gordon, K. D., Clayton, G. C., Misselt, K. A., Landolt, A. U., \& Wolff, M. J. 2003, ApJ, 594, 279

Gouiffes, C., Finley, J. P., \& Ögelman, H. 1992, ApJ, 394, 581

Gradari, S., Barbieri, M., Barbieri, C., et al. 2011, MNRAS, 412, 2689

Hewish, A., Bell, S. J., Pilkington, J. D. H., Scott, P. F., \& Collins, R. A. 1968, Natur, 217, 709

Hill, R. J., Dolan, J. F., Bless, R. C., et al. 1997, ApJL, 486, L99

Hobbs, G. B., Edwards, R. T., \& Manchester, R. N. 2006, MNRAS, 369, 655

Johnston, S., Romani, R. W., Marshall, F. E., \& Zhang, W. 2004, MNRAS, 355,31

Kaaret, P., Marshall, H. L., Aldcroft, T. L., et al. 2001, ApJ, 546, 1159

Kargaltsev, O., Klingler, N., Chastain, S., \& Pavlov, G. G. 2017, Journal of Physics: Conference Series, 932, 012050

Kargaltsev, O., \& Pavlov, G. G. 2007, Ap\&SS, 308, 287

Kargaltsev, O. Y., Pavlov, G. G., \& Romani, R. W. 2004, ApJ, 602, 372

Kargaltsev, O. Y., Pavlov, G. G., Zavlin, V. E., \& Romani, R. W. 2005, ApJ, 625,307

Lundqvist, N., Lundqvist, P., Björnsson, C.-I., et al. 2011, MNRAS, 413, 611

Maan, Y. 2015, ApJ, 815, 126

Manchester, R. N., Hobbs, G. B., Teoh, A., \& Hobbs, M. 2005, AJ, 129, 1993

Manchester, R. N., Mar, D. P., Lyne, A. G., et al. 1993, ApJL, 1993, L29

Marshall, F. E., Gotthelf, E. V., Zhang, W., Middleditch, J., \& Wang, Q. D. 1998, ApJ, 499, L179

Marshall, F. E., Guillemot, L., Harding, A. K., Martin, P., \& Smith, D. A. 2015, ApJL, 807, L27

Marshall, F. E., Guillemot, L., Harding, A. K., Martin, P., \& Smith, D. A. 2016, ApJL, 827, L39

Marshall, F. E., Guillemot, L., Harding, A. K., Martin, P., \& Smith, D. A. 2018, AAS Meeting Abstracts, 231, 243.05

Middleditch, J., \& Pennypacker, C. 1985, Natur, 313, 659

Middleditch, J., Pennypacker, C. R., \& Burns, M. S. 1987, ApJ, 315, 142

Mignani, R., De Luca, A., Caraveo, P. A., \& Becker, W. 2002, ApJL, 580, L47

Mignani, R. P. 2011, AdSpR, 47, 1281

Mignani, R. P., Bagnulo, S., de Luca, A., et al. 2007, Ap\&SS, 308, 203

Mignani, R. P., Caraveo, P. A., \& Bignami, G. F. 1998, A\&A, 332, L37

Mignani, R. P., De Luca, A., Hummel, W., et al. 2012, A\&A, 544, 100

Mignani, R. P., Paladino, R., Rudak, B., et al. 2017, ApJL, 851, L10

Mignani, R. P., Pavlov, G. G., \& Kargaltsev, O. 2010a, ApJ, 720, 1635

Mignani, R. P., Pulone, L., Iannicola, G., et al. 2005, A\&A, 431, 659

Mignani, R. P., Rea, N., Testa, V., et al. 2016a, MNRAS, 461, 4317

Mignani, R. P., Sartori, A., De Luca, A., et al. 2010b, A\&A, 515, 110
Mignani, R. P., Testa, V., Marelli, M., et al. 2016b, ApJ, 825, 151

Moos, H. W., Cash, W. C., Cowie, L. L., et al. 2000, ApJL, 538, L1

Moran, P., Mignani, R. P., Collins, S., et al. 2013, MNRAS, 436, 401

Naletto, G., Barbieri, C., Occhipinti, T., et al. 2009, A\&A, 508, 531

Nasuti, F. P., Mignani, R., Caraveo, P. A., \& Bignami, G. F. 1996, A\&A, 314,849

Pacini, F. 1968, Natur, 219, 145

Pavlov, G. G., Rangelov, B., Kargaltsev, O., et al. 2017, ApJ, 850, 79

Percival, J. W., Biggs, J. D., Dolan, J. F., et al. 1993, ApJ, 407, 276

Petre, R., Hwang, U., \& Holt, S. S. 2007, ApJ, 662, 988

Proffit, C. R. 2006, in The 2005 HST Calibration Workshop: Hubble After the Transition to Two-Gyro Mode, ed. A. M. Kroekmoer, P. Goudgrooij, \& L. L. Dressel (Baltimore: STScI), 234

Rangelov, B., Pavlov, G. G., Kargaltsev, O., et al. 2017, ApJ, 835, 264

Ray, P. S., Kerr, M., Parent, D., et al. 2011, ApJS, 194, 17

Riley, A. 2017, STIS Instrument Handbook, Version 16.0 (Baltimore: STScI)

Romani, R. W., Kargaltsev, O., \& Pavlov, G. G. 2005, ApJ, 627, 383

Sandberg, A., \& Sollerman, J. 2009, A\&A, 504, 525

Serafimovich, N. I., Shibanov, Y. A., Lundqvist, P., \& Sollerman, J. 2004, A\&A, 425, 1041

Seward, F. D., Harnden, F. R., Jr., \& Helfand, D. J. 1984, ApJL, 287, L19

Shearer, A., Golden, A., Harfst, S., et al. 1998, A\&A, 335, L21

Shearer, A., Redfern, M., Gorman, G., et al. 1997, ApJL, 487, L181

Shearer, A., Redfern, M., Pedersen, H., et al. 1994, ApJL, 423, L51

Shibanov, Y. A., Sollerman, J., Lundqvist, P., Gull, T., \& Lindler, D. 2005, A\&A, 440, 693

Skrutskie, M. F., Cutri, R. M., Stiening, R., et al. 2006, AJ, 131, 1163

Słowikowska, A., Kanbach, G., Borkowski, J., \& Becker, W. 2007, Neutron Stars and Pulsars 40 years After the Discovery, MPE-Report 291

Słowikowska, A., Kanbach, G., Kramer, M., \& Stefanescu, A. 2009, MNRAS, 397, 103

Sollerman, J. 2003, A\&A, 406, 639

Sollerman, J., Lundqvist, P., Lindler, D., et al. 2000, ApJ, 537, 861

Spolon, A., Zampieri, L., Burtovoi, A., et al. 2019, MNRAS, 482, 175

Standish, E. M. 1982, A\&A, 114, 297

Storm, J., Gieren, W., Forqué, P., et al. 2011, A\&A, 534, A95

Wallace, P. T., Peterson, B. A., Murdin, P. G., et al. 1977, Natur, 266, 692

Zharikov, S., \& Mignani, R. P. 2013, MNRAS, 435, 2227

Zyuzin, D., Shibanov, Y., Danilenko, A., Mennickent, R. E., \& Zharikov, S. 2013, ApJ, 775, 101 